\title{
STRUCTURAL PROPERTIES OF THE ONE-DIMENSIONAL DRIFT-DIFFUSION MODELS FOR SEMICONDUCTORS
}

\author{
FATIHA ALABAU
}

\begin{abstract}
This paper is devoted to the analysis of the one-dimensional current and voltage drift-diffusion models for arbitrary types of semiconductor devices and under the assumption of vanishing generation recombination. We show in the course of this paper, that these models satisfy structural properties, which are due to the particular form of the coupling of the involved systems. These structural properties allow us to prove an existence and uniqueness result for the solutions of the current driven model together with monotonicity properties with respect to the total current $I$, of the electron and hole current densities and of the electric field at the contacts. We also prove analytic dependence of the solutions on $I$. These results allow us to establish several qualitative properties of the current voltage characteristic. In particular, we give the nature of the (possible) bifurcation points of this curve, we show that the voltage function is an analytic function of the total current and we characterize the asymptotic behavior of the currents for large voltages. As a consequence, we show that the currents never saturate as the voltage goes to $\pm \infty$, contrary to what was predicted by numerical simulations by M. S. Mock (Compel. 1 (1982), pp. 165-174). We also analyze the drift-diffusion models under the assumption of quasi-neutral approximation. We show, in particular, that the reduced current driven model has at most one solution, but that it does not always have a solution. Then, we compare the full and the reduced voltage driven models and we show that, in general, the quasi-neutral approximation is not accurate for large voltages, even if no saturation phenomenon occurs. Finally, we prove a local existence and uniqueness result for the current driven model in the case of small generation recombination terms.
\end{abstract}

\section{INTRODUCTION}

In this paper, we consider the one-dimensional current driven and voltage driven drift-diffusion models. These models describe the transport of charges in a semiconductor device. They consist of a nonlinear system of differential equations which depends on a real parameter. This parameter is the applied bias across the device in the case of the voltage driven model, whereas it is the current flowing through the device in the case of the current driven model.

We assume that the semiconductor is not degenerate, so that the Einstein relations are valid. Moreover, we assume that there is no generation recombination. Under these assumptions and for a prescribed current $I$ in $\mathbb{R}$, the current driven

Received by the editors October 23, 1993 and, in revised form, October 31, 1994.

1991 Mathematics Subject Classification. Primary 35G30, 35J25, 35B50.

Key words and phrases. Semiconductor, electrochemistry, nonlinear system, elliptic, existence, uniqueness, monotonicity. 
drift-diffusion equations are, in scaled form (see [14], and [11] for the scaling):

$$
\begin{gathered}
\varepsilon \psi^{\prime \prime}=n-p-N \text { in } \Omega=(-1,1), \\
\mu_{n}\left(n^{\prime}-n \psi^{\prime}\right)=J_{n} \text { in } \Omega, \\
\mu_{p}\left(p^{\prime}+p \psi^{\prime}\right)=J_{n}-I \text { in } \Omega, \\
J_{n}^{\prime}=0 \text { in } \Omega,
\end{gathered}
$$

subject to the boundary conditions:

$$
\begin{aligned}
& \psi(-1)=\psi_{-1}, \text { where } \psi_{-1}=\log \left(\frac{N(-1)+\sqrt{N^{2}(-1)+4 \delta^{4}}}{2 \delta^{2}}\right), \\
& n(x)=n_{x}, \text { where } n_{x}=\frac{N(x)+\sqrt{N^{2}(x)+4 \delta^{4}}}{2}, \quad x= \pm 1 \\
& p(x)=p_{x}, \text { where } p_{x}=\frac{-N(x)+\sqrt{N^{2}(x)+4 \delta^{4}}}{2}, \quad x= \pm 1 .
\end{aligned}
$$

System (1.1)-(1.7) models the transport of electrons and holes in a semiconductor device. The scaled unknowns are the functions $\psi, n, p$ and the number $J_{n}$, which stand respectively for the electrostatic potential, the electron and hole densities and for the electron current density. The number $I-J_{n}$ and the function $-\psi^{\prime}$ represent respectively the hole current density and the electric field. The doping profile $N$ and the mobilities $\mu_{n}, \mu_{p}$, which are given functions defined on $\bar{\Omega}$, as well as the positive numbers $\varepsilon$ and $\delta$, depend only on the semiconductor device and not on the parameter $I$. It should be noted that in practice $\varepsilon$ and $\delta^{2}$ which stand respectively for the normed Debye length (the scaling factor being the size of the unscaled device) and the normed intrinsic concentration (the scaling factor being the $L^{\infty}$ norm of the unscaled doping profile) are small parameters.

We recall that the semiconductor is said to be symmetric, when $N$ is odd. The boundary data $n_{ \pm 1}, p_{ \pm 1}$ given in (1.6)-(1.7), correspond to ideal ohmic contacts. This means that they satisfy the following usual electro-neutrality condition

$$
n_{x}-p_{x}-N(x)=0, \quad x= \pm 1,
$$

and the following thermal equilibrium condition

$$
n_{-1} p_{-1}=n_{1} p_{1}=\delta^{4} .
$$

It is easy to check that the results presented in this paper are valid, with minor changes, for arbitrary boundary data $n_{ \pm 1}>0, p_{ \pm 1}>0$.

Since the nonlinear system (1.1)-(1.7) depends on the parameter $I$, it is denoted from now on by $(\mathrm{CD})_{I}$.

In the case of the one-dimensional current driven model, the bias $V$ across the device is unknown. It is defined by

$$
V=\log \left(\frac{N(1)+\sqrt{N^{2}(1)+4 \delta^{4}}}{2 \delta^{2}}\right)-\psi(1),
$$

where $\psi$ is the electrostatic potential corresponding to a solution of $(\mathrm{CD})_{I}$. We remark, that at this stage, (1.10) is only a formal definition, since existence and uniqueness of the solutions of the bipolar system $(\mathrm{CD})_{I}$ has only been proved for 
sufficiently small $|I|$ (see [10]). We recall that in the unipolar drift-diffusion model (which is valid for particular devices only) one of the carrier current is assumed to be zero, that is $I-J_{n}=0$ (resp. $J_{n}=0$ ) in case of conduction electrons (resp. holes). The unipolar case is of course simpler to analyze than the bipolar case, since the bipolar system $(\mathrm{CD})_{I}$ is reduced to a system of two equations only in the unipolar case. In this latter case existence for sufficiently small $|I|$ and uniqueness for arbitrary $I$ has been obtained in [10].

The voltage driven model is slightly different from the current driven one. Actually, in the case of the voltage driven model, the bias $V$ is given, whereas the current $I$ is unknown. Hence, for a given $V$ in $\mathbb{R}$, the voltage driven system is defined by (1.1)-(1.10), and

$$
I^{\prime}=0 \text { in } \Omega \text {, }
$$

where (1.10) gives the boundary condition on $\psi$ at the point $x=1$. The unknowns of this system are therefore $\psi, n, p, J_{n}$ and $I$. Since this system depends on the parameter $V$, it is denoted from now on by $(\mathrm{VD})_{V}$.

We recall that the voltage current characteristic expresses the unknown currents $I$ in terms of $V$. Moreover, in the framework of electrochemistry, the fact that $I$ (resp. $V$ ) is prescribed, means that we consider galvanostatic (resp. potentiostatic) conditions.

Because of physical considerations, the mobilities are positive functions. In this paper, we assume that the mobilities are either constant, or space dependent. In this last case, we assume in addition that the mobilities are bounded away from zero, that is

$$
\exists \mu>0 \text { such that } \mu \leq \mu_{n}(x), \mu \leq \mu_{p}(x) \quad \forall x \in \bar{\Omega} .
$$

We recall that the existence of weak solutions $\left(\psi, n, p, J_{n}, I\right)$ of $(\mathrm{VD})_{V}$ has been proved for arbitrary $V$ in $\mathbb{R}$ by many authors (see e.g [11] and the references therein). Moreover any solution $\left(\psi, n, p, J_{n}, I\right)$ of $(\mathrm{VD})_{V}$ satisfies

$$
0<n, 0<p \text {. }
$$

The current and voltage driven systems are related formally in the following way:

Assume that $I$ is given and let $\left(\psi, n, p, J_{n}\right)$ be a solution (if it exists) of $(\mathrm{CD})_{I}$, then $\left(\psi, n, p, J_{n}, I\right)$ is a solution of $(\mathrm{VD})_{V}$, where $V$ is given by (1.10). Conversely, assume that $V$ is given and let $\left(\psi, n, p, J_{n}, I\right)$ be a solution of $(\mathrm{VD})_{V}$. The equations (1.2) and (1.3) can be written under the form

$$
(n \exp (-\psi))^{\prime}=J_{n} \mu_{n}^{-1} \exp (-\psi),
$$

and

$$
(p \exp (\psi))^{\prime}=\left(J_{n}-I\right) \mu_{p}^{-1} \exp (\psi) .
$$

An integration from $x=-1$ to $x=1$ of these two last equations together with (1.4)-(1.10), give

$$
\begin{aligned}
I= & \delta^{2}(\exp (V)-1)\left(\int_{\Omega} \exp (-\psi) \mu_{n}^{-1} d x\right)^{-1} \\
& +\delta^{2}(1-\exp (-V))\left(\int_{\Omega} \exp (\psi) \mu_{p}^{-1} d x\right)^{-1} .
\end{aligned}
$$

Hence, $\left(\psi, n, p, J_{n}\right)$ is a solution of $(\mathrm{CD})_{I}$, where $I$ is given by (1.12).

Since the solutions of $(\mathrm{VD})_{V}$ are not unique in general (see [13], [17] for numerical examples), it is expected that $(\mathrm{CD})_{I}$ might have no solutions for certain values of 
$I$. On the other hand, it has been proved in $[10]$ that $(\mathrm{CD})_{I}$ has a unique solution for sufficiently small $|I|$ and for constant and equal mobilities. In [13], the author gives a numerical example of multiple solutions of $(\mathrm{VD})_{V}$. In this example the currents on the lower and middle branches of the voltage current characteristic, apparently saturate (i.e. reach a finite limit) as $V$ goes to $+\infty$, whereas the upper and middle branches connect through a turning point. Hence for this example, $(\mathrm{CD})_{I}$ has apparently no solutions for certain values of $I$. Recently, new numerical simulations (see [15], [17]) lead to the conjecture that the currents do not saturate and that the lower and middle branches should connect through another turning point. Therefore the questions of existence and uniqueness of solutions of $(\mathrm{CD})_{I}$ for a given $I$, are open.

The purpose of the present paper is to answer these questions and to establish additional qualitative properties of the solutions of the systems $(\mathrm{CD})_{I}$ and $(\mathrm{VD})_{V}$, under general assumptions on the doping profile $N$. At this stage, it is important to pinpoint in which mathematical and physical context our work takes place. We are interested in formal and general mathematical properties of the drift-diffusion equations. The results presented here are general in the sense that they are independent (from a mathematical point of view) on the physical scales of the device. They are formal in the sense that the physical models under consideration in this paper are simplified (one-dimensional case, ... ) and their physical validity broken down beyond a certain range of device parameters and doping densities. In the course of this paper, we show that both systems $(\mathrm{CD})_{I}$ and $(\mathrm{VD})_{V}$ possess structural properties, due to the specific form of the coupling between the two convection-diffusion equations (1.2), (1.3) and the Poisson equation (1.1). These properties are structural in the sense that they depend only on the structure of the involved systems, and not on the values of the physical data which are involved. Furthermore, these intrinsic properties lead to the qualitative properties which are given in this paper. We describe these structural properties in the remarks given next after Theorem 2.1 and after the proof of Theorem 2.7. Thanks to these properties, we first prove that, for any type of semiconductor devices, $(\mathrm{CD})_{I}$ has a unique weak solution for every $I$, which depends in an analytic way on $I$.

We then establish qualitative properties of the voltage current characteristic. In particular, we show that the voltage function is a well defined, analytic function of $I$ and that the (possible) bifurcation points of the voltage current characteristic are turning points. We also characterize the asymptotic behavior of the voltage current characteristic as $|V|$ goes to $+\infty$ by showing that the currents corresponding to $V$ satisfy

$$
|I|=\mathcal{O}(|V|) \quad \text { as } \quad|V| \rightarrow+\infty
$$

where $\mathcal{O}$ denotes the usual Landau symbol. This proves in particular, that the currents never saturate as $|V|$ goes to $+\infty$, so that the saturation phenomenon obtained in [13] was probably due to numerical effects.

We then establish qualitative properties of the solutions of $(\mathrm{VD})_{V}$ and $(\mathrm{CD})_{I}$ which are useful to questions related to the uniqueness of the solutions of $(\mathrm{VD})_{V}$. In particular, we first prove that if all the solutions of $(\mathrm{VD})_{V}$ are isolated for all $V \geq V_{c}^{+}$(or resp. all $V \leq V_{c}^{-}$), then (VD) $V$ has a unique solution for all $V \geq V_{c}^{+}$ (or resp. all $V \leq V_{c}^{-}$). Therefore, if the data $N, \mu_{n}, \mu_{p}, \varepsilon$ and $\delta$ are such that every solution of $(\mathrm{VD})_{V}$ is locally unique for all $V$, then $(\mathrm{VD})_{V}$ has a unique solution for all $V$. Thus, for the example of a $p n$ junction, for which it is expected that 
$(\mathrm{VD})_{V}$ has a unique solution for all $V$, the above result shows that it is sufficient to prove local uniqueness of all the solutions of $(\mathrm{VD})_{V}$. Moreover, using the structural property which leads to the above-mentioned uniqueness result, we prove that if the total current $I$ strictly increases, then the electron and hole currents together with the electric field at the ohmic contacts \pm 1 , strictly increase. We have already proved and used such monotonicity properties under restrictive assumptions on $N$ (as e.g. $N=0$ or $N=\operatorname{sign}(x)$ ) in [5], [2], [1]. The present paper shows that these properties hold under very general assumptions on $N$. Such properties are particularly useful for proving uniqueness results for $(\mathrm{VD})_{V}$ under additional hypotheses on $N$ (see the above-mentioned papers).

Then, we analyze the drift-diffusion models under the assumption of quasineutral approximation. In practice, $\varepsilon$ is a small parameter. Hence, both systems $(\mathrm{CD})_{I}$ and $(\mathrm{VD})_{V}$ can be studied in the framework of singular perturbation theory. We recall that the quasi-neutral approximation of $(\mathrm{CD})_{I}\left(\right.$ resp. $\left.(\mathrm{VD})_{V}\right)$ is obtained by setting $\varepsilon=0$ in (1.1) and by adding interface conditions at the points where $N$ is discontinuous. The resulting systems are respectively called the reduced current and voltage driven drift-diffusion models and are denoted respectively by $(\mathrm{RCD})_{I}$ and $(\mathrm{RVD})_{V}$, whereas the systems $(\mathrm{CD})_{I}$ and $(\mathrm{VD})_{V}$ are respectively called the full current and voltage driven models. Multiplicity results and numerical saturation phenomena for $(\mathrm{RVD})_{V}$ have been obtained in [15] and [16]. In this section, we first prove that the uniqueness result obtained for $(\mathrm{CD})_{I}$ is preserved when one considers $(\mathrm{RCD})_{I}$, but that the existence result is not preserved, in general, for $(\mathrm{RCD})_{I}$. In particular, we give, in the case of symmetric devices, a necessary and sufficient condition on $N$, for saturation of the reduced currents. If we denote respectively by $I$ and $\bar{I}$, the currents corresponding respectively to (VD) $V$ and (RVD) $V$, then it has been proved in [9], that for fixed $V$, the asymptotic error $|I-\bar{I}|$ goes to 0 as $\varepsilon$ goes to 0 . Under various restrictive assumptions on $N$ or on $V$, estimates of the form

$$
|I-\bar{I}| \leq c(V) \varepsilon^{r}, \text { with } r>0, c(V)>0,
$$

have been obtained in [12], [3], [4] for fixed $V$ as $\varepsilon$ goes to 0 . The purpose of section 4 of the present paper, is to show that in general the quasi-neutral approximation is not accurate for large $|V|$, this even if no saturation phenomenon occurs. This proves that in general, asymptotic error estimates like (1.13) cannot be uniform with respect to $V$.

Finally, we prove a local existence and uniqueness result for the current driven model in the case of small generation recombination terms.

Throughout this paper, we consider weak solutions of $(\mathrm{CD})_{I}$ and $(\mathrm{VD})_{V}$. Moreover, a function $f$ defined on $\bar{\Omega}$, is said to be piecewise continuous (resp. analytic) on $\bar{\Omega}$, if there exists $\left(a_{i}\right)_{1 \leq i \leq M} \in(\bar{\Omega})^{M}$, satisfying $a_{1}=-1<\ldots<a_{i}<a_{i+1}<$ $\ldots<a_{M}=1$, such that $f$ is continuous (resp. analytic) on each subintervals $\left[a_{i}, a_{i+1}\right]$.

The paper is organized as follows. In section 2 , we give the existence and uniqueness results for the current driven model. In section 3, we give the qualitative properties of the voltage current characteristic, and we establish the monotonicity properties of the electron and hole currents and of the electric field at the ohmic contacts. In section 4, we analyze the reduced current and voltage driven models. We give a general uniqueness result for the reduced current driven model, and we prove that the quasi-neutral approximation is not accurate, in general, for large 
$|V|$. We conclude in the last section, extend the existence and uniqueness result of section 2 to the case of small generation recombination terms, and indicate some of the open questions related to this paper.

\section{EXISTENCE AND UNIQUENESS RESUlts FOR THE CURRENT DRIVEN MODEL}

The main results of this section are the Uniqueness Theorem 2.1 and the Existence Theorem 2.7 for the current driven model. This section is organized as follows. We first give the Uniqueness Theorem and its proof. In the remark next after this proof, we describe more explicitly the structural property of $(\mathrm{CD})_{I}$, which leads to this uniqueness result. Then, we give the Existence Theorem. Before to give its proof, we establish several intermediate results, which give new a priori estimates on the solutions of the voltage driven model. The main intermediate results are Lemmas 2.8, 2.9 and 2.16. We first give Lemma 2.8 and its proof, then Lemma 2.9. Since the proof of Lemma 2.9 is very technical, we establish intermediate results in Propositions 2.10-2.15. Next, we give the proof of Lemma 2.9. Then, we give Lemma 2.16 and its proof, together with the proof of Theorem 2.7. In the remark next after this proof, we describe more explicitly the structural properties of $(\mathrm{VD})_{V}$, which lead to the existence theorem. We conclude this section by Corollary 2.17, which gives the conditions on the data which guarantee that $(\mathrm{CD})_{I}$ has a unique solution. We now give the uniqueness theorem.

Theorem 2.1. Assume that $N$ is in $L^{\infty}(\Omega)$ and that $\mu_{n}, \mu_{p}$ are piecewise continuous on $\bar{\Omega}$ and satisfy (1.11). Then for every $I$ in $\mathbb{R},(\mathrm{CD})_{I}$ has at most one weak solution $\left(\psi, n, p, J_{n}\right)$.

The proof of this result requires several steps. For the sake of clearness, we give these intermediate results in the form of propositions (together with their proofs) during the proof of Theorem 2.1.

Proof. Let $I$ be given such that there exist two weak solutions $\left(\psi, n, p, J_{n}\right)$ and $\left(\tilde{\psi}, \tilde{n}, \tilde{p}, \tilde{J}_{n}\right)$ of $(\mathrm{CD})_{I}$. It is easy to check that $(\psi-\tilde{\psi})$ is indeed in $\mathcal{C}^{2}(\bar{\Omega})$, and that $n-\tilde{n}$ and $p-\tilde{p}$ are in $\mathcal{C}(\bar{\Omega})$. We get from $(1.1)$

$$
\varepsilon(\psi-\tilde{\psi})^{\prime \prime}=(n-\tilde{n})-(p-\tilde{p}) \quad \text { in } \Omega .
$$

Moreover, since $\mu_{n}$ and $\mu_{p}$ are piecewise continuous on $\bar{\Omega}$ and satisfy (1.11), we deduce that $(n-\tilde{n}) \exp (-\psi)$ and $(p-\tilde{p}) \exp (\psi)$ are piecewise continuously differentiable on $\bar{\Omega}$. This implies that there exist $M$ points $\left(a_{i}\right)_{1 \leq i \leq M}$ in $(\bar{\Omega})^{M}$ satisfying

$$
a_{1}=-1<\ldots<a_{i}<a_{i+1}<\ldots<a_{M}=1 .
$$

and such that the restrictions of $(n-\tilde{n}) \exp (-\psi)$ and $(p-\tilde{p}) \exp (\psi)$ to each subinterval $\left[a_{i}, a_{i+1}\right]$ are in $\mathcal{C}^{1}\left(\left[a_{i}, a_{i+1}\right]\right)$. Hence, we deduce from (1.2) and (1.3) that $(n-\tilde{n}) \exp (-\psi)$ and $(p-\tilde{p}) \exp (\psi)$ satisfy respectively the following equations in each subinterval $\left[a_{i}, a_{i+1}\right]$ :

$$
((n-\tilde{n}) \exp (-\psi))^{\prime}=\tilde{n} \exp (-\psi)(\psi-\tilde{\psi})^{\prime}+\left(J_{n}-\tilde{J}_{n}\right) \mu_{n}^{-1} \exp (-\psi),
$$

and,

$$
((p-\tilde{p}) \exp (\psi))^{\prime}=-\tilde{p} \exp (\psi)(\psi-\tilde{\psi})^{\prime}+\left(J_{n}-\tilde{J}_{n}\right) \mu_{p}^{-1} \exp (\psi) .
$$

Now we remark from (1.4) that $J_{n}-\tilde{J}_{n}$ is a constant. Hence if $(\psi-\tilde{\psi})^{\prime}$ is identically zero on $\Omega$, then we deduce easily from (2.2) and (2.3) that the two solutions coincide 
on $\bar{\Omega}$. Therefore we can assume that $(\psi-\tilde{\psi})^{\prime}$ is not identically zero on $\Omega$. Moreover, since $J_{n}-\tilde{J}_{n}$ is a constant, we can assume without loss of generality that

$$
J_{n}-\tilde{J}_{n} \leq 0
$$

holds. We set

$$
X=\left\{x \in \bar{\Omega},(\psi-\tilde{\psi})^{\prime}(x)=0\right\} .
$$

Then we have the following intermediate result:

Proposition 2.2. Assume that (2.4) holds and that $(\psi-\tilde{\psi})^{\prime}$ is not identically zero on $\Omega$. Then $X \cap \Omega \neq \emptyset$.

Proof of Proposition 2.2. We proceed by contradiction. Assume to the contrary that $X \cap \Omega$ is empty. Since $(\psi-\tilde{\psi})$ is indeed in $\mathcal{C}^{2}(\bar{\Omega})$, we deduce that $(\psi-\tilde{\psi})^{\prime}$ keeps a constant sign in $\Omega$. If $(\psi-\tilde{\psi})^{\prime} \leq 0$ (resp. $\left.\geq 0\right)$ on $\Omega$, we deduce from $(2.2)$ (resp. (2.3)), (2.4) and from the boundary conditions $(n-\tilde{n})( \pm 1)=0$ (resp. $(p-\tilde{p})( \pm 1)=0)$ that

$$
n-\tilde{n}=0 \quad(\text { resp. } p-\tilde{p}=0) \quad \text { on } \bar{\Omega} .
$$

This, together with $(2.2)$ (resp. $(2.3))$ imply that $(\psi-\tilde{\psi})^{\prime}$ is identically zero on $\Omega$, which is impossible, from our hypotheses. Hence we proved that $X \cap \Omega$ is not empty. This concludes the proof of Proposition 2.2.

We now need the following result:

Proposition 2.3. Assume that (2.4) holds and that $(\psi-\tilde{\psi})^{\prime}$ is not identically zero on $\Omega$. Then the following inequality holds:

$$
J_{n}-\tilde{J}_{n}<0 .
$$

Proof of Proposition 2.3. We proceed by contradiction. Assume that (2.5) does not hold. Since (2.4) is verified, we get

$$
J_{n}-\tilde{J}_{n}=0 .
$$

Therefore $(n-\tilde{n}) \exp (-\psi)$ and $(p-\tilde{p}) \exp (\psi)$ are in $\mathcal{C}^{1}(\bar{\Omega})$. We claim now that $(\psi-\tilde{\psi})^{\prime}(-1)=0$ holds. In order to prove this assertion, let us assume to the contrary that $(\psi-\tilde{\psi})^{\prime}(-1) \neq 0$. From Proposition 2.2 we know that $X \cap \Omega$ is not empty, therefore there exists $x^{\star} \in X \cap \Omega$ such that $(\psi-\tilde{\psi})^{\prime}$ keeps a constant sign on $\left[-1, x^{\star}\right]$ and is not identically zero on this interval. Assume first that $(\psi-\tilde{\psi})^{\prime} \leq 0$ on $\left[-1, x^{\star}\right]$. Then we deduce from $(2.6),(2.2),(2.3)$ and the boundary conditions $(n-\tilde{n})(-1)=(p-\tilde{p})(-1)=0$ that

$$
(n-\tilde{n})\left(x^{\star}\right)<0, \quad 0<(p-\tilde{p})\left(x^{\star}\right) .
$$

Setting now $x=x^{\star}$ in (2.1) and using (2.7), we obtain the inequality $(\psi-\tilde{\psi})^{\prime \prime}\left(x^{\star}\right)<$ 0 . But this is impossible since $x^{\star}$ is in $X \cap \Omega$ and since $(\psi-\tilde{\psi})^{\prime} \leq 0$ on $\left[-1, x^{\star}\right]$.

The case where $(\psi-\tilde{\psi})^{\prime} \geq 0$ on $\left[-1, x^{\star}\right]$ leads, in a similar way, to a contradiction. Hence we proved that if $(2.6)$ is satisfied, then $(\psi-\tilde{\psi})^{\prime}(-1)=0$ holds. We now set

$$
U=\left(\psi-\tilde{\psi},(\psi-\tilde{\psi})^{\prime},(n-\tilde{n}) \exp (-\psi),(p-\tilde{p}) \exp (\psi)\right)^{T}
$$

Then $U$ is a solution of the following linear initial value problem:

$$
W^{\prime}(x)=A(x) W(x), \quad \forall x \in \Omega, W(-1)=0,
$$


where the function $A$ is continuous from $\bar{\Omega}$ to $\mathbb{R}^{4,4}$. Since $A$ is continuous, this initial value problem has a unique solution on $\bar{\Omega}$, which is the trivial solution. Therefore $U$ is identically zero on $\bar{\Omega}$. But this contradicts one of the hypotheses of Proposition 2.3. Hence we proved that if (2.5) does not hold then both cases $(\psi-\tilde{\psi})^{\prime}(-1)=0$ and $(\psi-\tilde{\psi})^{\prime}(-1) \neq 0$ lead to a contradiction. This proves that (2.5) necessarily holds, which concludes the proof of Proposition 2.3.

Hence, we can assume from now on, that (2.5) holds. Since $X$ is not empty, we can define

$$
\alpha=\inf (X), \beta=\sup (X) .
$$

Moreover, since $(\psi-\tilde{\psi})^{\prime}$ is continuous on $\bar{\Omega}, X$ is a closed subset of $\bar{\Omega}$. Hence $\alpha$ and $\beta$ are in $X$. In addition, since $X \cap \Omega$ is not empty, we have

$$
\alpha<1,-1<\beta \text {. }
$$

We now need the following intermediate result:

Proposition 2.4. Assume that (2.4) holds. Then the following inequalities holds:

$$
(n-\tilde{n})(\alpha) \leq 0,(p-\tilde{p})(\alpha) \leq 0,
$$

and,

$$
(n-\tilde{n})(\beta) \geq 0,(p-\tilde{p})(\beta) \geq 0 .
$$

Moreover if $\alpha \neq-1$ (resp. $\beta \neq 1$ ) holds, then both inequalities of (2.9) (resp. of (2.10)) are strict inequalities.

Proof of Proposition 2.4. If $\alpha=-1$ (or resp. $\beta=1$ ) holds, then (2.9) (or resp. (2.10)) trivially holds. Assume now that $\alpha \neq-1$ and $\beta \neq 1$. Hence, since (2.8) holds, we can assume that $\alpha$ and $\beta$ are in $\Omega$. This implies in particular that the points -1 and 1 are not in $X$. Therefore $(\psi-\tilde{\psi})^{\prime}$ keeps a constant sign on $[-1, \alpha]$ and $[\beta, 1]$ and is not identically zero on these two intervals. We now first prove that (2.9) holds with strict inequalities. We proceed as follows:

Since $(\psi-\tilde{\psi})^{\prime}$ keeps a constant sign on $[-1, \alpha]$, we have either $(\psi-\tilde{\psi})^{\prime} \leq 0$ or $(\psi-\tilde{\psi})^{\prime} \geq 0$ on $[-1, \alpha]$.

Assume first that $(\psi-\tilde{\psi})^{\prime} \leq 0$ on $[-1, \alpha]$. Then, since $(\psi-\tilde{\psi})^{\prime}$ is not identically zero on $[-1, \alpha]$, we deduce from $(2.2),(2.4)$ and from the boundary condition $(n-\tilde{n})(-1)=0$ that

$$
(n-\tilde{n})(\alpha)<0 .
$$

Two subcases can now occur: either $(\psi-\tilde{\psi})^{\prime \prime}(\alpha)=0$ or $(\psi-\tilde{\psi})^{\prime \prime}(\alpha) \neq 0$. If the first subcase occurs, then we deduce from (2.1) and (2.11) that

$$
(p-\tilde{p})(\alpha)<0
$$

so that $(2.9)$ holds with strict inequalities. Assume now that $(\psi-\tilde{\psi})^{\prime \prime}(\alpha) \neq 0$. Since $\alpha$ is in $X$, this implies that $(\psi-\tilde{\psi})^{\prime}$ changes sign at $x=\alpha$. But we have assumed that $(\psi-\tilde{\psi})^{\prime} \leq 0$ on $[-1, \alpha]$, therefore $(\psi-\tilde{\psi})$ has a local minimum at $x=\alpha$. This together with (2.1) and (2.11) imply that

$$
(p-\tilde{p})(\alpha)<0
$$

Hence we proved that if $(\psi-\tilde{\psi})^{\prime} \leq 0$ on $[-1, \alpha]$, then for both subcases $(\psi-\tilde{\psi})^{\prime \prime}(\alpha)=$ 0 or $(\psi-\tilde{\psi})^{\prime \prime}(\alpha) \neq 0,(2.9)$ holds with strict inequalities. 
Assume now that $(\psi-\tilde{\psi})^{\prime} \geq 0$ on $[-1, \alpha]$. Then we proceed as for the former case, but we use (2.3) instead of $(2.2)$, and the boundary condition $(p-\tilde{p})(-1)=0$, instead of $(n-\tilde{n})(-1)=0$. This leads to

$$
(p-\tilde{p})(\alpha)<0 \text {. }
$$

We then prove that either $(\psi-\tilde{\psi})^{\prime \prime}(\alpha)=0$ or that $(\psi-\tilde{\psi})$ has a local maximum at $x=\alpha$. Using (2.1) once again, we deduce that for both subcases (2.9) holds with strict inequalities. Hence we finally proved that if $\alpha \neq-1$ holds, then (2.9) holds with strict inequalities.

We prove in a similar way that if $\beta \neq 1$ holds, then (2.10) holds with strict inequalities (in this case, we consider the interval $[\beta, 1]$, and we use the appropriate equation (2.2) or (2.3) and the appropriate boundary condition $(n-\tilde{n})(1)=0$ or $(p-\tilde{p})(1)=0$, depending on the sign of $(\psi-\tilde{\psi})^{\prime}$ on $\left.[\beta, 1]\right)$. This concludes the proof of Proposition 2.4.

We claim now that $\alpha=\beta$ holds. Assume to the contrary that $\alpha<\beta$ holds. We set

$$
Y=\{x \in X-[-1, \alpha], 0 \leq(n-\tilde{n})(x) \text { or } 0 \leq(p-\tilde{p})(x)\} .
$$

Since (2.10) and $\alpha<\beta$ hold, we deduce that $\beta$ is in $Y$. Therefore we can define $\gamma=\inf (Y)$. Since $n-\tilde{n}$ and $p-\tilde{p}$ are continuous on $\bar{\Omega}$, we deduce that

$$
(n-\tilde{n})(\gamma) \geq 0 \quad \text { or } \quad(p-\tilde{p})(\gamma) \geq 0 .
$$

Moreover we have, by definition of $\gamma$

$$
\alpha \leq \gamma, \quad \gamma \in X .
$$

On the other hand, since $\alpha$ is in $X$ and since $(n-\tilde{n}) \exp (-\psi)$ and $(p-\tilde{p}) \exp (\psi)$ are piecewise continuously differentiable on $\bar{\Omega}$, we deduce from (2.5), and from (2.2) and (2.3) respectively that

$$
\lim _{h \rightarrow 0^{+}}((n-\tilde{n}) \exp (-\psi))^{\prime}(\alpha+h)<0 \text { and } \lim _{h \rightarrow 0^{+}}((p-\tilde{p}) \exp (\psi))^{\prime}(\alpha+h)<0 .
$$

This, together with (2.9) imply that

(2.13) $\exists \eta>0$ such that $(n-\tilde{n})(x)<0,(p-\tilde{p})(x)<0 \quad \forall x \in(\alpha, \alpha+\eta]$.

Since $\alpha$ does not belong to $Y$ and since (2.13) holds, we get

$$
\alpha<\gamma \text {. }
$$

We now need the following intermediate result:

Proposition 2.5. Assume that (2.4) and that $\alpha<\min (\beta, \gamma)$ hold. If the following property holds:

$$
\exists z \in X \cap[\alpha, \gamma) \text { such that }(n-\tilde{n})(z) \leq 0,(p-\tilde{p})(z) \leq 0,
$$

then $X \cap(z, \gamma) \neq \emptyset$.

Proof of Proposition 2.5. Assume that (2.15) holds. In order to prove the proposition, we proceed by contradiction. Assume that $X \cap(z, \gamma)=\emptyset$. Then $(\psi-\tilde{\psi})^{\prime}$ keeps a constant sign in $(z, \gamma)$ and does not vanish on this interval.

Assume first that $(\psi-\tilde{\psi})^{\prime} \leq 0$ in $(z, \gamma)$. Then, since $(\psi-\tilde{\psi})^{\prime}$ is not identically zero on $[-1, \alpha]$, we deduce from $(2.2),(2.15)$ and $(2.4)$ that

$$
(n-\tilde{n})(\gamma)<0 \text {. }
$$


This, together with the boundary condition $(n-\tilde{n})(1)=0,(2.1)$ and (2.12) imply that $\gamma<1$ and that $(\psi-\tilde{\psi})^{\prime \prime}(\gamma) \neq 0$. Moreover using (2.14), we have $-1<\gamma$. Therefore, since $\gamma$ is in $X \cap \Omega$, we deduce that $(\psi-\tilde{\psi})^{\prime}$ changes sign at $x=\gamma$. Hence, since we have assumed that $(\psi-\tilde{\psi})^{\prime} \leq 0$ in $(z, \gamma),(\psi-\tilde{\psi})$ has a local minimum at $x=\gamma$. This, together with (2.1) imply that

$$
(p-\tilde{p})(\gamma) \leq(n-\tilde{n})(\gamma) .
$$

But since $(n-\tilde{n})(\gamma)<0$ already holds, we deduce that $(p-\tilde{p})(\gamma)<0$. This contradicts (2.12). Therefore we proved that the case where $(\psi-\tilde{\psi})^{\prime} \leq 0$ in $(z, \gamma)$ is impossible. The case where $(\psi-\tilde{\psi})^{\prime} \geq 0$ in $(z, \gamma)$ leads in a similar way to a contradiction (in this case, we use $(2.3)$ instead of $(2.2)$ and prove that $(\psi-\tilde{\psi})$ has a local maximum at $x=\gamma)$.

Hence, we proved that if $(z, \gamma) \cap X=\emptyset$, then both cases $(\psi-\tilde{\psi})^{\prime} \leq 0$ or $(\psi-\tilde{\psi})^{\prime} \geq 0$ in $(z, \gamma)$ lead to a contradiction. This proves that $(z, \gamma) \cap X \neq \emptyset$ and concludes the proof of Proposition 2.5.

We now need the following last intermediate result:

Proposition 2.6. Assume that (2.4), and that $\alpha<\min (\beta, \gamma)$ hold. Then there exists a sequence $\left(z_{i}\right)_{i \in \mathbb{N}}$ of points of $(\alpha, \gamma) \cap X$ which satisfies the following properties:

$$
(n-\tilde{n})\left(z_{i}\right)<0,(p-\tilde{p})\left(z_{i}\right)<0 \quad \forall i \in \mathbb{N},
$$

and

$$
\lim _{i \mapsto+\infty} z_{i}=\gamma .
$$

Proof of Proposition 2.6. Since (2.9) and $\alpha<\gamma$ hold, the point $z=\alpha$ satisfies (2.15). Using Proposition 2.5, we deduce that there exists $\bar{z} \in(\alpha, \gamma) \cap X$. Therefore, by definition of $\gamma, \bar{z}$ does not belong to $Y$. Hence, since $\bar{z} \in X-[-1, \alpha]$, we deduce that we have

$$
(n-\tilde{n})(\bar{z})<0,(p-\tilde{p})(\bar{z})<0 .
$$

Therefore, the set

$$
Z=\{z \in X \cap(\alpha, \gamma),(n-\tilde{n})(z)<0,(p-\tilde{p})(z)<0\}
$$

is not empty, so that we can define $l=\sup (Z)$. Of course, since $X$ is closed and since $\bar{z} \in X \cap(\alpha, \gamma)$, we have $l \in X \cap(\alpha, \gamma]$. Moreover, by definition of $l$, and since $n-\tilde{n}$ and $p-\tilde{p}$ are continuous on $\bar{\Omega}$, we deduce that

$$
(n-\tilde{n})(l) \leq 0,(p-\tilde{p})(l) \leq 0 .
$$

We claim that $l=\gamma$ holds. Assume to the contrary that $l<\gamma$ holds. Therefore, since (2.18) holds and since $l \in X \cap(\alpha, \gamma)$, we deduce that $z=l$ satisfies (2.15). Using Proposition 2.5, we deduce that there exists $z^{\star} \in X \cap(l, \gamma)$. By definition of $\gamma, z^{\star}$ does not belong to $Y$. Since $z^{\star} \in X-[-1, \alpha]$, we deduce that $z^{\star} \in Z$. But this is impossible, since $l=\sup (Z)$ and $l<z^{\star}$ hold. Hence, we proved that $l=\gamma$. Now, since (2.12) holds, we deduce that $\gamma=l$ does not belong to $Z$. Therefore, by definition of $l$, we deduce that there exists a sequence $\left(z_{i}\right)_{i \in \mathbb{N}}$ of points of $Z$, such that (2.17) holds. This proves (2.16)-(2.17) and concludes the proof of Proposition 2.6.

We can now conclude the proof of Theorem 2.1. Assume that $\alpha<\beta$ holds. Then, since (2.14) holds, we deduce from Proposition 2.6 that there exists a sequence 
$\left(z_{i}\right)_{i \in \mathbb{N}}$ of points of $Z$ satisfying (2.16) and (2.17). Moreover, since $l=\gamma$ and (2.18) hold, we have

$$
(n-\tilde{n})(\gamma) \leq 0,(p-\tilde{p})(\gamma) \leq 0 .
$$

Using these last inequalities together with (2.12), we get

$$
(n-\tilde{n})(\gamma)=0 \text { or }(p-\tilde{p})(\gamma)=0 .
$$

Let us first assume that $(p-\tilde{p})(\gamma)=0$. Since $\gamma$ is in $X$, we deduce from (2.3) and (2.5) that

$$
\lim _{h \rightarrow 0^{-}}((p-\tilde{p}) \exp (\psi))^{\prime}(\gamma+h)<0 .
$$

This, together with $(p-\tilde{p})(\gamma)=0$, imply that there exists $\eta>0$ such that

$$
0<p-\tilde{p} \text { in }[\gamma-\eta, \gamma) .
$$

But this contradicts (2.16)-(2.17). In a similar way, we prove that if $(n-\tilde{n})(\gamma)=0$, then there exists $\eta>0$, such that $0<n-\tilde{n}$ in $[\gamma-\eta, \gamma)$, which is impossible since (2.16)-(2.17) hold.

Therefore, we finally proved our claim, that is: if (2.5) holds, then $\alpha=\beta$. By definition of $\alpha$ and $\beta$, we deduce that $X=\{\alpha\}$. Moreover, since (2.8) holds, we deduce that $\alpha$ is in $\Omega$. Using now Proposition 2.4, and since $\alpha \neq-1$ and $\beta \neq 1$, we deduce that

$$
(n-\tilde{n})(\alpha)<0,(p-\tilde{p})(\alpha)<0,
$$

whereas,

$$
(n-\tilde{n})(\beta)>0,(p-\tilde{p})(\beta)>0 .
$$

But this is impossible, since $\alpha=\beta$ holds. Hence, we proved that the case where (2.5) holds, leads to a contradiction. This, together with Proposition 2.3, concludes the proof of Theorem 2.1.

Remark. In the case where $N, \mu_{n}, \mu_{p}$ are piecewise analytic in $\bar{\Omega}$, we can give a simpler proof of Theorem 2.1. Moreover, in this case, we can describe more explicitly the structural property which leads to the above uniqueness theorem. We keep the notation of the proof of Theorem 2.1 and we assume, without loss of generality, that (2.4) holds. This simpler proof and this description are as follows:

Since the data are assumed to be piecewise analytic, it follows from classical results on differential equations (see e.g. [7]) that $(\psi-\tilde{\psi})^{\prime}$ is piecewise analytic in $\bar{\Omega}$. Therefore we can define the set $\bar{Z}$ of points of $\Omega$ at which $(\psi-\tilde{\psi})^{\prime}$ changes sign. Of course this set is finite, and if $(\psi-\tilde{\psi})^{\prime}$ keeps a constant sign on $\Omega$, then this set is empty. Assume first that $\bar{Z}$ is not empty and denote by $\left(z_{i}\right)_{1 \leq i \leq K}$ its elements ordered in a strictly increasing sequence. By definition $(\psi-\tilde{\psi})^{\prime}$ keeps a constant sign on the intervals $\left[-1, z_{1}\right],\left[z_{i}, z_{i+1}\right]$ and $\left[z_{K}, 1\right]$. As in the proof of Theorem 2.1, we show that if $(\psi-\tilde{\psi})^{\prime} \leq 0$ (resp. $\left.\geq 0\right)$ on $\left[-1, z_{1}\right]$, then by using only properties of the convection-diffusion equation (1.2) (resp. (1.3)), we have

$$
(n-\tilde{n})\left(z_{1}\right)<0 \quad\left(\text { resp. }(p-\tilde{p})\left(z_{1}\right)<0\right) .
$$

At this stage, the specific form of the coupling between (1.2), (1.3) and (1.1) has not been used. We can show now, how this specific coupling leads to the desired result. By definition, the points $z_{i}$ are extrema of the function $(\psi-\tilde{\psi})$. Since this function is in $\mathcal{C}^{2}(\bar{\Omega})$, we deduce that for each $z_{i}$, we have either $0 \leq(\psi-\tilde{\psi})^{\prime \prime}\left(z_{i}\right)$ 
or $0 \geq(\psi-\tilde{\psi})^{\prime \prime}\left(z_{i}\right)$. Now, using (1.1) independently of (1.2) and (1.3), we deduce that

$$
\begin{aligned}
& \text { if }(\psi-\tilde{\psi})^{\prime} \leq 0(\text { resp. } \geq 0) \text { on }\left[z_{i-1}, z_{i}\right]\left(\text { with } z_{0}=-1\right) \\
& \text { then we have } \forall i \in\{1, \ldots, K\} \text {, }
\end{aligned}
$$$$
(p-\tilde{p})\left(z_{i}\right) \leq(n-\tilde{n})\left(z_{i}\right)\left(\operatorname{resp} .(p-\tilde{p})\left(z_{i}\right) \geq(n-\tilde{n})\left(z_{i}\right)\right) .
$$

Therefore, we derived (2.19) by using only the properties of the convection-diffusion equations (1.2) and (1.3), whereas we derived (2.20) by using only the Poisson equation (1.1). Now, we can use the coupling between (1.1) and (1.2), (1.3), by setting $i=1$ in (2.20) and by using (2.19). From this, we deduce that

$$
(n-\tilde{n})\left(z_{1}\right)<0,(p-\tilde{p})\left(z_{1}\right)<0 .
$$

In a similar way, using the properties of the appropriate equation (1.2) or (1.3), depending on the sign of $(\psi-\tilde{\psi})^{\prime}$ on the intervals $\left[z_{i-1}, z_{i}\right]$ and $(2.20)$, we prove by induction on $i$, starting from $x=-1$ that the following inequalities hold:

$$
(n-\tilde{n})\left(z_{i}\right)<0,(p-\tilde{p})\left(z_{i}\right)<0 \quad \forall i \in\{1, \ldots, K\} .
$$

But since $(\psi-\tilde{\psi})^{\prime}$ keeps a constant sign on $\left[z_{K}, 1\right]$ and using the properties of the appropriate equation (1.2) or (1.3), we deduce that

$$
0>(n-\tilde{n})(1), \quad 0>(p-\tilde{p})(1)
$$

also holds. But this is impossible, since (2.22) contradicts the boundary conditions $(n-\tilde{n})(1)=(p-\tilde{p})(1)=0$. This proves that the set $\bar{Z}$ is necessarily empty, which means that $(\psi-\tilde{\psi})^{\prime}$ keeps a constant sign on $\bar{\Omega}$. Then we deduce easily that the two solutions coincide on $\bar{\Omega}$. In the case where the data are no longer assumed to be piecewise analytic, we can no longer define the set $\bar{Z}$. This is the reason why we need a more refined proof, which is still based on the same structural property which has been described above. It is interesting to remark that this property also leads to the Monotonicity Lemmas 3.11 and 3.12 .

Theorem 2.7. Assume that $N$ is $L^{\infty}(\Omega)$ and that $\mu_{n}, \mu_{p}$ are strictly positive constants. Then for every $I$ in $\mathbb{R},(\mathrm{CD})_{I}$ has at least one weak solution $\left(\psi, n, p, J_{n}\right)$.

In order to prove this theorem we consider the voltage driven problem $(\mathrm{VD})_{V}$. For a given solution $\left(\psi, n, p, J_{n}, I\right)$ of $(\mathrm{VD})_{V}$, we define two unknown constants $I_{V}$ and $J_{V}$ as follows:

$$
I_{V}=J_{n} \mu_{n}^{-1}+\left(I-J_{n}\right) \mu_{p}^{-1}, \quad J_{V}=J_{n} \mu_{n}^{-1}-\left(I-J_{n}\right) \mu_{p}^{-1} .
$$

Before giving the proof of Theorem 2.7, we establish new a priori estimates on the solutions of $(\mathrm{VD})_{V}$, which are given in Lemmas 2.8, 2.9 and 2.16. Moreover, in order to establish Lemma 2.9, we also need intermediate results which are given in Propositions 2.10, 2.11, 2.12, 2.13, 2.14 and 2.15. We now give all these intermediate results.

Lemma 2.8. Assume that the hypotheses of Theorem 2.7 hold and let $V$ be given in $\mathbb{R}$. Then for any solution $\left(\psi, n, p, J_{n}, I\right)$ of $(\mathrm{VD})_{V}$, the following property holds:

If the unknown constant $I_{V} J_{V}$ satisfies

$$
I_{V} J_{V} \leq 0
$$

then the following estimate holds:

$$
(n-p)(x) \leq N_{+} \quad \forall x \in \bar{\Omega} .
$$


If the unknown constant $I_{V} J_{V}$ satisfies

$$
I_{V} J_{V} \geq 0
$$

then the following estimate holds:

$$
N_{-} \leq(n-p)(x) \quad \forall x \in \bar{\Omega},
$$

where $I_{V}$ and $J_{V}$ are defined by (2.23) and where the numbers $N_{-}$and $N_{+}$are given by

$$
N_{-}=\min \left(\inf _{x \in \bar{\Omega}}(N(x)), 0\right), N_{+}=\max \left(\sup _{x \in \bar{\Omega}}(N(x)), 0\right)
$$

and where inf and sup stand respectively for the essential inf and the essential sup.

Proof. Let $\left(\psi, n, p, J_{n}, I\right)$ be a weak solution of $(\mathrm{VD})_{V}$, then $(\psi, n, p)$ is in $\left(H^{2}(\Omega)\right)^{3}$. Dividing (1.2) and (1.3) respectively by $\mu_{n}$ and $\mu_{p}$ and adding the two resulting equations, we get on $\bar{\Omega}$

$$
(n-p)^{\prime}=(n+p) \psi^{\prime}+I_{V},
$$

where $I_{V}$ is defined by the first equation of (2.23). Therefore $n-p$ is a weak solution of

$$
(n-p)^{\prime \prime}=(n+p) \psi^{\prime \prime}+(n+p)^{\prime} \psi^{\prime} .
$$

Dividing now (1.2) and (1.3) respectively by $\mu_{n}$ and $\mu_{p}$ and subtracting the two resulting equations, we obtain on $\bar{\Omega}$

$$
(n+p)^{\prime}=(n-p) \psi^{\prime}+J_{V},
$$

where $J_{V}$ is defined by the second equation of (2.23). Substituing this last equation in (2.28), multiplying the resulting equation by $\varepsilon$, and using (1.1), we deduce that $n-p$ is a weak solution of

$$
\varepsilon(n-p)^{\prime \prime}-(n-p)\left(\varepsilon \psi^{\prime 2}+n+p\right)=-N(n+p)+\varepsilon \psi^{\prime} J_{V} .
$$

Using now the expression of $\psi^{\prime}$ given by (2.27) in (2.30), we deduce that $n-p$ is a weak solution of

$$
\begin{aligned}
& \varepsilon(n-p)^{\prime \prime}-\varepsilon J_{V}(n-p)^{\prime}(n+p)^{-1}-(n-p)\left(\varepsilon \psi^{2}+n+p\right) \\
& \quad=-N(n+p)-\varepsilon I_{V} J_{V}(n+p)^{-1} .
\end{aligned}
$$

Now assume first that (i) holds. We set $u=n-p-N_{+}$, where $N_{+}$is defined in (2.26). Then $u$ is a weak solution of

$$
\mathcal{L} u=N_{+} \varepsilon \psi^{\prime 2}+\left(N_{+}-N\right)(n+p)-\varepsilon I_{V} J_{V}(n+p)^{-1},
$$

where $\mathcal{L}$ is the second order linear differential operator defined by

$$
\mathcal{L} v=\varepsilon v^{\prime \prime}-\varepsilon J_{V}(n+p)^{-1} v^{\prime}-\left(\varepsilon \psi^{2}+n+p\right) v .
$$

By definition of $N_{+}$and since $(n+p)>0$ and (i) hold, we deduce from (2.32) that $0 \leq \mathcal{L} u$ holds in a weak sense. Now, since $(n+p)>0$ holds, we can apply the weak maximum principle (see [8]) to $\mathcal{L}$. This, together with the fact that $n-p \in \mathcal{C}(\bar{\Omega})$, implies that $u \leq 0$ on $\bar{\Omega}$, so that (2.24) holds. In a similar way, we prove that if (ii) holds, then (2.25) holds. This concludes the proof of the lemma. 
Lemma 2.9. Assume that the hypotheses of Theorem 2.7 hold. Then there exist $V_{2}^{+} \geq 0$ and two constants $c_{1}^{+}>0$ and $c_{2}^{+}$such that for all $V \geq V_{2}^{+}$, every solution $\left(\psi, n, p, J_{n}, I\right)$ of $(\mathrm{VD})_{V}$ satisfies

$$
c_{1}^{+} V+c_{2}^{+} \leq I
$$

In order to prove Lemma 2.9, we need to consider both cases (i) and (ii) of Lemma 2.8. But as will be seen in the sequel, several subcases of (i) and (ii) have also to be considered. For the sake of clearness, these different subcases are treated in the Propositions 2.10, 2.11, 2.12, 2.13, 2.14, 2.15, whose proofs are given below. In order to simplify our presentation, we use the following notations for the proof of the Propositions 2.10, 2.11, 2.12, 2.13, 2.14, and 2.15, as well as for the proof of Lemma 2.9 .

If $\left(\psi, n, p, J_{n}, I\right)$ is a solution of (VD) $V$ such that case (i) (resp. (ii)) of Lemma 2.8 holds, we define a number $x_{e}^{+}$and a function $d_{+}$by setting

$$
x_{e}^{+}=1 \quad\left(\operatorname{resp} . x_{e}^{+}=-1\right),
$$

and,

$$
d_{+}(x)=n(x) \quad\left(\text { resp. } d_{+}(x)=p(x)\right) \quad \forall x \in \bar{\Omega} .
$$

Moreover, any solution $\left(\psi, n, p, J_{n}, I\right)$ of $(\mathrm{VD})_{V}$ satisfies either one of the following two inequalities:

$$
0 \leq n^{\prime}\left(x_{e}^{+}\right) \quad \text { or } \quad p^{\prime}\left(x_{e}^{+}\right) \leq 0
$$

or it satisfies the following two inequalities:

$$
n^{\prime}\left(x_{e}^{+}\right)<0 \quad \text { and } \quad 0<p^{\prime}\left(x_{e}^{+}\right) .
$$

The case where (2.36) (resp. (2.37)) holds is treated in Proposition 2.11 (resp. Propositions 2.13, 2.14, and 2.15).

If $\left(\psi, n, p, J_{n}, I\right)$ is a solution of $(\mathrm{VD})_{V}$ such that $(2.37)$ holds, then we have in particular $(n-p)^{\prime}\left(x_{e}^{+}\right)<0$. Since $(n-p)$ is in $\mathcal{C}^{1}(\bar{\Omega})$, such a solution satisfies either

$$
(n-p)^{\prime} \leq 0 \quad \text { on } \bar{\Omega}
$$

or,

$$
\exists x \in \Omega \quad \text { such that } \quad 0<(n-p)^{\prime}(x) .
$$

In both cases (2.38) or (2.39), there exists $x_{m}^{+}$in $\bar{\Omega}$ such that the following properties hold:

$$
x_{m}^{+} \neq x_{e}^{+}
$$

and,

$$
(n-p)^{\prime}(x) \leq 0 \quad \forall x \in\left[\min \left(x_{m}^{+}, x_{e}^{+}\right), \max \left(x_{m}^{+}, x_{e}^{+}\right)\right] .
$$

Moreover, if (2.38) (resp. (2.39)) holds, we can choose $x_{m}^{+}$such that

$$
x_{m}^{+}=-x_{e}^{+} \quad\left(\operatorname{resp} .(n-p)^{\prime}\left(x_{m}^{+}\right)=0\right) .
$$

Hence if (2.37) holds, it is always possible to choose $x_{m}^{+}$such that (2.40), (2.41) and (2.42) hold.

We now give the different propositions (together with their proofs) which allow us to prove Lemma 2.9. Using the above notations we have the following results: 
Proposition 2.10. Assume that the hypotheses of Theorem 2.7 hold and let $V \geq 0$ be given. Then any solution $\left(\psi, n, p, J_{n}, I\right)$ of $(\mathrm{VD})_{V}$ satisfies

$$
0 \leq J_{n}, \quad 0 \leq I-J_{n}, \quad 0 \leq I_{V},
$$

and,

$$
\psi^{\prime}\left(x_{e}^{+}\right) \leq \frac{1}{2}\left(U_{b i}-V+\frac{2\left(N_{+}-N_{-}\right)}{\varepsilon}\right),
$$

where $I_{V}$ and $x_{e}^{+}$are respectively defined in (2.23) and (2.34) and where $U_{b i}$ is given by

$$
U_{b i}=\log \left(\frac{N(1)+\sqrt{N^{2}(1)+4 \delta^{4}}}{N(-1)+\sqrt{N^{2}(-1)+4 \delta^{4}}}\right) .
$$

Proof. Let $V \geq 0$ be given and let $\left(\psi, n, p, J_{n}, I\right)$ be an arbitrary solution of $(\mathrm{VD})_{V}$. From (1.12) it is clear that $I \geq 0$ holds. Moreover, the integration of (1.2) and (1.3) from $x=-1$ to $x=1$, together with the use of the boundary conditions (1.5)-(1.10) lead to the two last inequalities of (2.43).

The sign of the constant $J_{V}$ (defined by the second inequality of $(2.23)$ ) is unknown, hence we have to consider both cases (i) and (ii) of Lemma 2.8.

Let us first assume that (i) holds. Therefore from Lemma 2.8 we deduce that (2.24) is satisfied. From (2.24) and (1.1) we have

$$
\psi^{\prime \prime}(x) \leq\left(N_{+}-N_{-}\right) \varepsilon^{-1} \quad \text { for a.e. } x \in \Omega .
$$

We integrate this inequality from $x$ to 1 . This gives

$$
\psi^{\prime}(1) \leq \psi^{\prime}(x)+\left(N_{+}-N_{-}\right) \varepsilon^{-1}(1-x) \quad \forall x \in \bar{\Omega} .
$$

Integration of this last inequality from -1 to 1 gives

$$
2 \psi^{\prime}(1) \leq \psi(1)-\psi(-1)+2\left(N_{+}-N_{-}\right) \varepsilon^{-1} .
$$

This, together with the boundary conditions (1.5) and (1.10), gives (2.44) with $x_{e}^{+}=1$.

Assume now that (ii) holds, so that from Lemma 2.8, (2.25) is satisfied. From (2.25) and (1.1), we deduce that

$$
\psi^{\prime \prime}(x) \geq-\left(N_{+}-N_{-}\right) \varepsilon^{-1} \quad \text { for a.e. } x \in \Omega .
$$

Integration of this inequality from -1 to $x$ gives

$$
\psi^{\prime}(-1) \leq \psi^{\prime}(x)+\left(N_{+}-N_{-}\right) \varepsilon^{-1}(1+x) \quad \forall x \in \bar{\Omega} .
$$

We now integrate this last inequality from -1 to 1 . This gives

$$
2 \psi^{\prime}(-1) \leq \psi(1)-\psi(-1)+2\left(N_{+}-N_{-}\right) \varepsilon^{-1} .
$$

This, together with the boundary conditions (1.5) and (1.10), gives (2.44) with $x_{e}^{+}=-1$ and concludes the proof of the proposition.

Proposition 2.11. Assume that the hypotheses of Theorem 2.7 hold. Then there exists $V_{1}^{+} \geq 0$ defined by (2.61), such that, for all $V \geq V_{1}^{+}$and for every solution $\left(\psi, n, p, J_{n}, I\right)$ of $(\mathrm{VD})_{V}$ which satisfies (2.36), the following estimate holds:

$$
2^{-1} \mu_{-} k_{-}\left[V-\left(U_{b i}+2\left(N_{+}-N_{-}\right) / \varepsilon\right)\right] \leq I,
$$

where $U_{b i}$ is defined by (2.45) and where $\mu_{-}$and $k_{-}$are respectively given by

$$
\mu_{-}=\min \left(\mu_{n}, \mu_{p}\right)>0,
$$




$$
k_{-}=\min \left(n_{-1}, n_{1}, p_{-1}, p_{1}\right)>0 .
$$

Proof. Let $V \geq 0$ be given and let $\left(\psi, n, p, J_{n}, I\right)$ be a solution of $(\mathrm{VD})_{V}$ such that (2.36) holds.

Let us first assume that the first inequality of (2.36) holds. We set $x=x_{e}^{+}$in (1.2) and use (2.44). This gives

$$
2^{-1} \mu_{n} n\left(x_{e}^{+}\right)\left[V-\left(U_{b i}+2\left(N_{+}-N_{-}\right) \varepsilon^{-1}\right)\right] \leq J_{n} .
$$

From the second inequality of (2.43), we deduce that (2.46) holds for $V \geq V_{1}^{+}$, with $\mu_{-}$and $k_{-}$given respectively by (2.47) and (2.48).

Assume now that the second inequality of (2.36) holds. We set $x=x_{e}^{+}$in (1.3) and use (2.44). This gives

$$
2^{-1} \mu_{p} p\left(x_{e}^{+}\right)\left[V-\left(U_{b i}+2\left(N_{+}-N_{-}\right) \varepsilon^{-1}\right)\right] \leq I-J_{n} .
$$

From the first inequality of (2.43), we deduce that (2.46) also holds for $V \geq V_{1}^{+}$. This concludes the proof of the proposition.

Proposition 2.12. Assume that the hypotheses of Theorem 2.7 hold. Then for all $V \geq 0$ and for every solution $\left(\psi, n, p, J_{n}, I\right)$ of $(\mathrm{VD})_{V}$ satisfying (2.37), the following estimate holds:

$$
\psi^{\prime}(x) \leq \frac{1}{2}\left(-V+U_{b i}+6\left(N_{+}-N_{-}\right) / \varepsilon\right) \quad \forall x \in\left[\min \left(x_{m}^{+}, x_{e}^{+}\right), \max \left(x_{m}^{+}, x_{e}^{+}\right)\right],
$$

where $x_{e}^{+}$is defined as in (2.34) and where $x_{m}^{+}$is chosen such that (2.40), (2.41) and (2.42) hold.

Proof. Let $V \geq 0$ be given and let $\left(\psi, n, p, J_{n}, I\right)$ be a solution of $(\mathrm{VD})_{V}$ such that (2.37) holds. By our choice of $x_{m}^{+},(2.41)$ holds. Therefore we have for all $x$ in $\left[\min \left(x_{m}^{+}, x_{e}^{+}\right), \max \left(x_{m}^{+}, x_{e}^{+}\right)\right]$

$$
(n-p)\left(\max \left(x_{e}^{+}, x_{m}^{+}\right)\right) \leq(n-p)(x) \leq(n-p)\left(\min \left(x_{e}^{+}, x_{m}^{+}\right)\right) .
$$

Assume first that $x_{e}^{+}=1$, then we get from the left hand side of (2.50) and from $(1.1)$

$$
\psi^{\prime \prime}(x) \geq-\left(N_{+}-N_{-}\right) \varepsilon^{-1} \quad \text { for a.e. } x \in\left[x_{m}^{+}, 1\right],
$$

which in turn gives

$$
\psi^{\prime}(x) \leq \psi^{\prime}(1)+2\left(N_{+}-N_{-}\right) \varepsilon^{-1} \quad \forall x \in\left[x_{m}^{+}, 1\right] .
$$

This, together with (2.44) gives (2.49).

Assume now that $x_{e}^{+}=-1$, then we get from the right hand side of (2.50) and from $(1.1)$

$$
\psi^{\prime \prime}(x) \leq\left(N_{+}-N_{-}\right) \varepsilon^{-1} \quad \text { for a.e. } x \in\left[-1, x_{m}^{+}\right] .
$$

This, together with (2.44) gives (2.49), which concludes the proof of the proposition.

Proposition 2.13. Assume that the hypotheses of Theorem 2.7 hold and let $V_{2}^{+} \geq$ 0 be defined by (2.62). Assume moreover that $\left(\psi, n, p, J_{n}, I\right)$ is a solution of $(\mathrm{VD})_{V}$ with $V \geq V_{2}^{+}$, such that (2.37) holds. We define $x_{e}^{+}$and the function $d_{+}$respectively by (2.34) and (2.35), whereas $x_{m}^{+}$is chosen as inProposition 2.12. Then if $d_{+}^{\prime}$ changes sign in $\left(\min \left(x_{m}^{+}, x_{e}^{+}\right), \max \left(x_{m}^{+}, x_{e}^{+}\right)\right)$, the following estimate holds:

$$
2^{-1} \mu_{-} \min \left(n_{1}, p_{-1}\right)\left[V-\left(U_{b i}+6\left(N_{+}-N_{-}\right) / \varepsilon\right)\right] \leq I,
$$

where $\mu_{-}$is defined by (2.47). 
Proof. Let $V \geq V_{2}^{+}$be given and let $\left(\psi, n, p, J_{n}, I\right)$ be a solution of $(\mathrm{VD})_{V}$ such that (2.37) holds and such that $d_{+}^{\prime}$ changes sign in $\left(\min \left(x_{m}^{+}, x_{e}^{+}\right), \max \left(x_{m}^{+}, x_{e}^{+}\right)\right)$.

Assume first that $x_{e}^{+}=1$, then from (2.35), we have $d_{+}=n$. Since (2.37) holds, we have $n^{\prime}(1)<0$. By hypothesis, $n^{\prime}$ changes sign in $\left(x_{m}^{+}, 1\right)$, moreover $n$ is in $\mathcal{C}^{1}(\bar{\Omega})$. Therefore there exists $y_{m}^{+}$in $\left(x_{m}^{+}, 1\right)$ such that

$$
n^{\prime} \leq 0 \quad \text { on }\left[y_{m}^{+}, 1\right], \quad \text { and } \quad n^{\prime}\left(y_{m}^{+}\right)=0 .
$$

From (1.2) and the equality $n^{\prime}\left(y_{m}^{+}\right)=0$, we deduce that

$$
J_{n}=-\mu_{n} n\left(y_{m}^{+}\right) \psi^{\prime}\left(y_{m}^{+}\right) .
$$

Now since $y_{m}^{+}$is in $\left(x_{m}^{+}, 1\right)$, we can set $x=y_{m}^{+}$in (2.49). The resulting inequality, together with the inequality $\mu_{n} n\left(y_{m}^{+}\right)>0$ imply

$$
2^{-1} \mu_{n} n\left(y_{m}^{+}\right)\left[V-\left(U_{b i}+6\left(N_{+}-N_{-}\right) \varepsilon^{-1}\right)\right] \leq J_{n} .
$$

On the other hand, since we have $n^{\prime} \leq 0$ on $\left[y_{m}^{+}, 1\right]$, we get $n_{1} \leq n\left(y_{m}^{+}\right)$. This, together with (2.52), the second inequality of (2.43) and the inequality $V \geq V_{2}^{+}$ leads to (2.51).

Assume now that $x_{e}^{+}=-1$, then from (2.35), we have $d_{+}=p$. Since (2.37) holds, we have $p^{\prime}(-1)>0$. By hypothesis, $p^{\prime}$ changes sign in $\left(-1, x_{m}^{+}\right)$, moreover $p$ is in $\mathcal{C}^{1}(\bar{\Omega})$. Therefore there exists $z_{m}^{+}$in $\left(-1, x_{m}^{+}\right)$such that

$$
p^{\prime} \geq 0 \quad \text { on }\left[-1, z_{m}^{+}\right], \quad \text { and } \quad p^{\prime}\left(z_{m}^{+}\right)=0 .
$$

From (1.3) and the equality $p^{\prime}\left(z_{m}^{+}\right)=0$, we deduce that

$$
I-J_{n}=-\mu_{p} p\left(z_{m}^{+}\right) \psi^{\prime}\left(z_{m}^{+}\right) .
$$

As for the case $x_{e}^{+}=1$, we can set $x=z_{m}^{+}$in (2.49). The resulting inequality, together with the inequality $\mu_{p} p\left(z_{m}^{+}\right)>0$ implies

$$
2^{-1} \mu_{p} p\left(z_{m}^{+}\right)\left[V-\left(U_{b i}+6\left(N_{+}-N_{-}\right) \varepsilon^{-1}\right)\right] \leq I-J_{n} .
$$

On the other hand, since we have $p^{\prime} \geq 0$ on $\left[-1, z_{m}^{+}\right]$, we get $p_{-1} \leq p\left(z_{m}^{+}\right)$. This, together with (2.53), the first inequality of (2.43) and the inequality $V \geq V_{2}^{+}$leads to (2.51). This concludes the proof of the proposition.

The only case which remains now is the case where (2.37) holds and where $d_{+}^{\prime}$ keeps a constant sign in $\left[\min \left(x_{m}^{+}, x_{e}^{+}\right), \max \left(x_{m}^{+}, x_{e}^{+}\right)\right]$. For this last case, we have to consider the two subcases given respectively by (2.38) and (2.39). These two subcases are treated respectively in the next two propositions.

Proposition 2.14. Assume that the hypotheses of Theorem 2.7 hold and let $V_{2}^{+}$be defined by (2.62). For $V \geq V_{2}^{+}$, let $\left(\psi, n, p, J_{n}, I\right)$ be a solution of $(\mathrm{VD})_{V}$, such that (2.37) holds and such that $d_{+}^{\prime}$ keeps a constant sign in $\left[\min \left(x_{m}^{+}, x_{e}^{+}\right), \max \left(x_{m}^{+}, x_{e}^{+}\right)\right]$. Then if (2.38) holds, the following estimate is satisfied:

$$
2^{-1} \mu_{-}\left[\min \left(n_{1}, p_{-1}\right)\left(V-U_{b i}\right)-\left(N_{+}-N_{-}\right)\right] \leq I,
$$

where $\mu_{-}$is defined by (2.47).

Proof. Let $V \geq V_{2}^{+}$be given and assume that $\left(\psi, n, p, J_{n}, I\right)$ is a solution of $(\mathrm{VD})_{V}$ such that $(2.37)$ holds and such that $d_{+}^{\prime}$ keeps a constant sign in $\left[\min \left(x_{m}^{+}, x_{e}^{+}\right)\right.$, $\left.\max \left(x_{m}^{+}, x_{e}^{+}\right)\right]$. If $(2.38)$ holds, we have from $(2.42) x_{m}^{+}=-x_{e}^{+}$. Since $x_{e}^{+}$is in $\{-1,1\}$, we get

$$
\min \left(x_{m}^{+}, x_{e}^{+}\right)=-1, \max \left(x_{m}^{+}, x_{e}^{+}\right)=1
$$


Assume first that $x_{e}^{+}=1$, then from (2.35), we have $d_{+}=n$. Since (2.37) holds and since $d_{+}^{\prime}$ keeps a constant sign in $[-1,1]$, we have

$$
n_{1} \leq n(x) \quad \forall x \in[-1,1] .
$$

Assume now that $x_{e}^{+}=-1$, then from (2.35), we have $d_{+}=p$. Since (2.37) holds and since $d_{+}^{\prime}$ keeps a constant sign in $[-1,1]$, we have

$$
p_{-1} \leq p(x) \quad \forall x \in[-1,1] .
$$

Now since we have $n>0$ and $p>0$ in $[-1,1]$, we deduce that for both cases $x_{e}^{+}= \pm 1$, the following inequality holds:

$$
\min \left(n_{1}, p_{-1}\right) \leq(n+p)(x) \quad \forall x \in[-1,1] .
$$

Now since $x_{m}^{+}=-x_{e}^{+}$and since $x_{e}^{+}= \pm 1$, the inequality (2.49) holds for all $x$ in $[-1,1]$. This implies in particular that for $V \geq V_{2}^{+}$, the following inequality holds:

$$
\psi^{\prime}(x) \leq 0 \quad \forall x \in[-1,1] .
$$

From (1.2) and (1.3), we get

$$
(n-p)^{\prime}(x)=(n+p)(x) \psi^{\prime}(x)+J_{n} \mu_{n}^{-1}+\left(I-J_{n}\right) \mu_{p}^{-1} \quad \forall x \in[-1,1] .
$$

Using (2.55) and (2.56) in this last equality and integrating the resulting inequality from $x=-1$ to $x=1$, we obtain

$$
-\left(N_{+}-N_{-}\right) \leq \min \left(n_{1}, p_{-1}\right)\left(U_{b i}-V\right)+2\left(J_{n} \mu_{n}^{-1}+\left(I-J_{n}\right) \mu_{p}^{-1}\right) .
$$

We finally get (2.54) from the above inequality and from (2.43). This concludes the proof of the proposition.

We now consider the case where (2.39) holds.

Proposition 2.15. Assume that the hypotheses of Theorem 2.7 hold and let $V_{2}^{+}$be defined by (2.62). For $V \geq V_{2}^{+}$, let $\left(\psi, n, p, J_{n}, I\right)$ be a solution of $(\mathrm{VD})_{V}$, such that (2.37) holds and such that $d_{+}^{\prime}$ keeps a constant sign in $\left[\min \left(x_{m}^{+}, x_{e}^{+}\right), \max \left(x_{m}^{+}, x_{e}^{+}\right)\right]$. Then if (2.39) holds, the following estimate is satisfied:

$$
2^{-1} \mu_{-} \min \left(n_{1}, p_{-1}\right)\left[V-\left(U_{b i}+6\left(N_{+}-N_{-}\right) / \varepsilon\right)\right] \leq I,
$$

where $\mu_{-}$is defined by (2.47).

Proof. Let $V \geq V_{2}^{+}$be given and assume that $\left(\psi, n, p, J_{n}, I\right)$ is a solution of (VD) $V$ such that $(2.37)$ holds and such that $d_{+}^{\prime}$ keeps a constant sign in $\left[\min \left(x_{m}^{+}, x_{e}^{+}\right)\right.$, $\left.\max \left(x_{m}^{+}, x_{e}^{+}\right)\right]$. From this, we deduce as in the proof of Proposition 2.14 that

$$
\min \left(n_{1}, p_{-1}\right) \leq(n+p)\left(x_{m}^{+}\right) .
$$

Since (2.39) is assumed to hold, we have from (2.42)

$$
(n-p)^{\prime}\left(x_{m}^{+}\right)=0 \text {. }
$$

Setting $x=x_{m}^{+}$in (1.2) and (1.3) and using the above equality, we get

$$
J_{n} \mu_{n}^{-1}+\left(I-J_{n}\right) \mu_{p}^{-1}=-(n+p)\left(x_{m}^{+}\right) \psi^{\prime}\left(x_{m}^{+}\right) .
$$

We now set $x=x_{m}^{+}$in (2.49) and use the resulting inequality in (2.60). This gives

$$
2^{-1}(n+p)\left(x_{m}^{+}\right)\left[V-\left(U_{b i}+6\left(N_{+}-N_{-}\right) \varepsilon^{-1}\right)\right] \leq J_{n} \mu_{n}^{-1}+\left(I-J_{n}\right) \mu_{p}^{-1} .
$$

Using now (2.59) and (2.43) and the inequality $V \geq V_{2}^{+}$in this last inequality, we finally get (2.58). This concludes the proof of the proposition. 
We are now able to prove Lemma 2.9 .

Proof of Lemma 2.9. We define $V_{1}^{+}$and $V_{2}^{+}$respectively by

$$
V_{1}^{+}=\max \left(0, U_{b i}+2\left(N_{+}-N_{-}\right) / \varepsilon\right),
$$

and,

$$
V_{2}^{+}=\max \left(0, U_{b i}+6\left(N_{+}-N_{-}\right) / \varepsilon\right) .
$$

Let $V \geq V_{2}^{+}$be given and let $\left(\psi, n, p, J_{n}, I\right)$ be a solution of $(\mathrm{VD})_{V}$. Then this solution satisfies either (2.36) or (2.37). Assume first that this solution satisfies (2.36). Since $V_{2}^{+} \geq V_{1}^{+}$, we deduce from Proposition 2.11 that (2.46) holds. Assume now that (2.37) holds. Let $x_{e}^{+}, d_{+}$, and $x_{m}^{+}$be defined as above. Then either $d_{+}^{\prime}$ changes sign in $\left(\min \left(x_{m}^{+}, x_{e}^{+}\right), \max \left(x_{m}^{+}, x_{e}^{+}\right)\right)$or it keeps a constant sign in this interval. If $d_{+}^{\prime}$ changes sign in $\left(\min \left(x_{m}^{+}, x_{e}^{+}\right), \max \left(x_{m}^{+}, x_{e}^{+}\right)\right)$, we deduce from Proposition 2.13 and since $V \geq V_{2}^{+}$holds, that (2.51) is satisfied. If $d_{+}^{\prime}$ keeps a constant sign in $\left[\min \left(x_{m}^{+}, x_{e}^{+}\right), \max \left(x_{m}^{+}, x_{e}^{+}\right)\right]$, two subcases can occur: either (2.38) or (2.39) holds. If (2.38) holds, we use Proposition 2.14. We deduce that since $V \geq V_{2}^{+}$holds, (2.54) is satisfied. If (2.39) holds, we use Proposition 2.15. We deduce that since $V \geq V_{2}^{+}$holds, (2.58) is satisfied.

From all these cases we deduce that (2.33) holds with $V_{2}^{+}$given by (2.62), and $c_{1}^{+}>0$ and $c_{2}^{+}$given respectively by

$$
c_{1}^{+}=2^{-1} \mu_{-} \min \left(n_{1}, n_{-1}, p_{1}, p_{-1}\right),
$$

and,

$$
\begin{aligned}
c_{2}^{+}= & -2^{-1} \mu_{-}\left[\min \left(n_{1}, n_{-1}, p_{1}, p_{-1}\right) U_{b i}\right. \\
& \left.+\left(N_{+}-N_{-}\right) \varepsilon^{-1} \max \left(\varepsilon, 6 \min \left(n_{1}, p_{-1}\right)\right)\right] .
\end{aligned}
$$

This concludes the proof of the lemma.

We now consider the case where $V \leq 0$ holds. We obtain in this case the following lemma, which is analogous to Lemma 2.9 .

Lemma 2.16. Assume that the hypotheses of Theorem 2.7 hold. Then there exist $V_{2}^{-} \leq 0$ and two constants $c_{1}^{-}>0$ and $c_{2}^{-}$such that for all $V \leq V_{2}^{-}$, every solution $\left(\psi, n, p, J_{n}, I\right)$ of $(\mathrm{VD})_{V}$ satisfies

$$
c_{1}^{-} V+c_{2}^{-} \geq I
$$

The proof of this lemma is similar to that of Lemma 2.9, provided that some changes are taken into account. First, we now use the following notation:

If $\left(\psi, n, p, J_{n}, I\right)$ is a solution of (VD) $V$ such that case (i) (resp. (ii)) of Lemma 2.8 holds, we define a number $x_{e}^{-}$and a function $d_{-}$by setting

$$
x_{e}^{-}=-1 \quad\left(\text { resp. } x_{e}^{-}=1\right),
$$

and,

$$
d_{-}(x)=n(x) \quad\left(\text { resp. } d_{-}(x)=p(x)\right) \quad \forall x \in \bar{\Omega} .
$$

Moreover any solution $\left(\psi, n, p, J_{n}, I\right)$ of $(\mathrm{VD})_{V}$ satisfies either one of the following two inequalities:

$$
n^{\prime}\left(x_{e}^{-}\right) \leq 0 \quad \text { or } \quad 0 \leq p^{\prime}\left(x_{e}^{-}\right)
$$


or it satisfies the following two inequalities:

$$
0<n^{\prime}\left(x_{e}^{-}\right) \quad \text { and } \quad p^{\prime}\left(x_{e}^{-}\right)<0 .
$$

If (2.69) holds, then we have in particular $0<(n-p)^{\prime}\left(x_{e}^{-}\right)$. Hence if $\left(\psi, n, p, J_{n}, I\right)$ is a solution of $(\mathrm{VD})_{V}$ such that (2.69) holds, then this solution satisfies either

$$
0 \leq(n-p)^{\prime} \quad \text { on } \bar{\Omega},
$$

or,

$$
\exists x \in \Omega \quad \text { such that } \quad(n-p)^{\prime}(x)<0 .
$$

In both cases, there exists $x_{m}^{-}$in $\bar{\Omega}$ such that the following properties hold:

$$
x_{m}^{-} \neq x_{e}^{-},
$$

and,

$$
(n-p)^{\prime}(x) \geq 0 \quad \forall x \in\left[\min \left(x_{m}^{-}, x_{e}^{-}\right), \max \left(x_{m}^{-}, x_{e}^{-}\right)\right] .
$$

Moreover, if (2.70) (resp. (2.71)) holds, we can choose $x_{m}^{-}$such that

$$
x_{m}^{-}=-x_{e}^{-} \quad\left(\operatorname{resp} .(n-p)^{\prime}\left(x_{m}^{-}\right)=0\right) .
$$

Hence if (2.69) holds, it is always possible to choose $x_{m}^{-}$such that (2.72), (2.73) and (2.74) hold.

Proof of Lemma 2.16. We first remark that if $V \leq 0$ holds, then a proof similar to that of Proposition 2.10 shows that any solution $\left(\psi, n, p, J_{n}, I\right)$ of $(\mathrm{VD})_{V}$ satisfies

$$
I \leq 0, I-J_{n} \leq 0, I_{V} \leq 0,
$$

and,

$$
2^{-1}\left(-V+U_{b i}-2\left(N_{+}-N_{-}\right) \varepsilon^{-1}\right) \leq \psi^{\prime}\left(x_{e}^{-}\right),
$$

where $I_{V}$ is defined by the first equality of (2.23). We define $V_{1}^{-}$and $V_{2}^{-}$respectively by

$$
V_{1}^{-}=\min \left(0, U_{b i}-2\left(N_{+}-N_{-}\right) \varepsilon^{-1}\right),
$$

and,

$$
V_{2}^{-}=\min \left(0, U_{b i}-6\left(N_{+}-N_{-}\right) \varepsilon^{-1}\right) .
$$

Let $V \leq V_{2}^{-}$be given and let $\left(\psi, n, p, J_{n}, I\right)$ be a solution of $(\mathrm{VD})_{V}$. Then this solution satisfies either (2.68) or (2.69). Assume first that this solution satisfies (2.68). Then a proof similar to that of Proposition 2.11 shows that since $V \leq V_{1}^{-}$, the following estimate holds:

$$
I \leq 2^{-1} \mu_{-} k_{-}\left[V-\left(U_{b i}-2\left(N_{+}-N_{-}\right) \varepsilon^{-1}\right)\right]
$$

where $\mu_{-}$and $k_{-}$are respectively defined by (2.47) and (2.48). Assume now that $\left(\psi, n, p, J_{n}, I\right)$ is a solution of $(\mathrm{VD})_{V}$ such that (2.69) holds. Then a proof similar to that of Proposition 2.12 shows that the following estimate holds:

$$
2^{-1}\left(-V+U_{b i}-6\left(N_{+}-N_{-}\right) \varepsilon^{-1}\right) \leq \psi^{\prime}(x) \quad \forall x \in\left[\min \left(x_{m}^{-}, x_{e}^{-}\right), \max \left(x_{m}^{-}, x_{e}^{-}\right)\right]
$$

Two subcases can now occur as for the case $V \geq 0$ : either $d_{-}^{\prime}$ changes sign in $\left(\min \left(x_{m}^{-}, x_{e}^{-}\right), \max \left(x_{m}^{-}, x_{e}^{-}\right)\right)$or it keeps a constant sign in this interval. If $d_{-}^{\prime}$ changes sign in $\left(\min \left(x_{m}^{-}, x_{e}^{-}\right), \max \left(x_{m}^{-}, x_{e}^{-}\right)\right)$, then a proof similar to that of Proposition 2.13 shows that, since we have $V \leq V_{2}^{-}$, the following estimate holds:

$$
I \leq 2^{-1}\left(\mu_{-} \min \left(n_{-1}, p_{1}\right)\right)\left[V-\left(U_{b i}-6\left(N_{+}-N_{-}\right) / \varepsilon\right)\right],
$$


where $\mu_{-}$is defined by (2.47). If $d_{-}^{\prime}$ keeps a constant sign in $\left[\min \left(x_{m}^{-}, x_{e}^{-}\right)\right.$, $\left.\max \left(x_{m}^{-}, x_{e}^{-}\right)\right]$, two subcases can occur: either $(2.70)$ or $(2.71)$ holds. If $(2.70)$ holds, then a proof similar to that of Proposition 2.14 shows that since $V \leq V_{2}^{-}$ holds, the following estimate is satisfied:

$$
I \leq 2^{-1} \mu_{-}\left[\min \left(n_{-1}, p_{1}\right)\left(V-\left(U_{b i}\right)+\left(N_{+}-N_{-}\right)\right],\right.
$$

where $\mu_{-}$is defined by (2.47). If (2.71) holds, then a proof similar to that of Proposition 2.15 shows that since $V \leq V_{2}^{-}$holds, the following estimate is satisfied:

$$
\left.I \leq 2^{-1} \mu_{-} \min \left(n_{-1}, p_{1}\right)\left[V-\left(U_{b i}\right)-6\left(N_{+}-N_{-}\right) / \varepsilon\right)\right],
$$

where $\mu_{-}$is defined by (2.47).

From all these cases we deduce that (2.65) holds with $V_{2}^{-}$given by (2.76), and $c_{1}^{-}>0$ and $c_{2}^{-}$given respectively by

$$
c_{1}^{-}=c_{1}^{+}
$$

where $c_{1}^{+}$is given by $(2.63)$ and,

$$
\begin{aligned}
c_{2}^{-}= & -2^{-1} \mu_{-}\left[\min \left(n_{1}, n_{-1}, p_{1}, p_{-1}\right) U_{b i}\right. \\
& \left.+-\left(N_{+}-N_{-}\right) \varepsilon^{-1} \max \left(\varepsilon, 6 \min \left(n_{-1}, p_{1}\right)\right)\right] .
\end{aligned}
$$

This concludes the proof of the lemma.

We can now prove Theorem 2.7.

Proof of Theorem 2.7. We set

$$
\begin{aligned}
\mathcal{S}=\left\{\left(\psi, n, p, J_{n}, I, V\right) \in\left(H^{1}(\Omega)\right)^{3} \times \mathbb{R}^{3},\right. & \\
\left(\psi, n, p, J_{n}, I\right) & \text { is a weak solution of } \left.(V D)_{V}\right\}
\end{aligned}
$$

We now remark that $I$, defined as in (1.12), is a continuous function of $(\psi, n, p, V)$ in $\left(H^{1}(\Omega)\right)^{3} \times \mathbb{R}$. This, together with theorem 3.5.1 in [11] imply that $\mathcal{S}$ contains a connected subset $\mathcal{B}$ in $\left(H^{1}(\Omega)\right)^{3} \times \mathbb{R}^{3}$, which passes through the equilibrium solution $\left(\psi_{e}, n_{e}, p_{e}, 0,0,0\right)$ and whose projection on the $V$-axis equals $\mathbb{R}$. Since $\mathcal{B}$ is connected, its projection on the $I$-axis is an interval of $\mathbb{R}$. On the other hand, we deduce from Lemma 2.9 (resp. 2.16) that any solution $\left(\psi, n, p, J_{n}, I\right)$ of $(\mathrm{VD})_{V}$ satisfies

$$
I \rightarrow+\infty \quad(\text { resp. } \quad-\infty) \text { as } V \rightarrow+\infty \quad(\text { resp. } \quad-\infty)
$$

This implies in particular that the projection of $\mathcal{B}$ on the $I$-axis equals $\mathbb{R}$. This concludes the proof of Theorem 2.7.

Remark. The purpose of this remark is to describe more precisely the structural properties of $(\mathrm{VD})_{V}$ which lead to the Existence Theorem 2.7. If $\left(\psi, n, p, J_{n}, I\right)$ is a solution of $(\mathrm{VD})_{V}$, then $(n-p)(x)$ represents the mobile charge at $x$, whereas $N(x)$ represents the fixed charge at $x$. Lemma 2.8 shows that, if $\left(\psi, n, p, J_{n}, I\right)$ is a solution of $(\mathrm{VD})_{V}$, then, according to the sign of the unknown constant $I_{V} J_{V}$ and for a fixed $V$, the mobile charge is either bounded above or below, by a constant which depends only on the fixed charge. At this stage, we already used the coupling of the equations in $(\mathrm{VD})_{V}$ in order to derive equation (2.31), which in turn, leads to the result of Lemma 2.8. This lemma is essential for the obtaining of the a priori estimates on the currents $I$ given in Lemmas 2.9 and 2.16. Once we have the a priori estimate on the mobile charge, we show by using the Poisson equation (1.1), that the absolute value of the electric field at the contacts, is bounded below by 
a constant which depends linearly on the absolute value of the voltage. We show in Propositions 2.11-2.15, how this last estimate combined with properties of the convection-diffusion equations (1.2)-(1.3), lead to the estimate of Lemma 2.9. In a similar way, we obtain the estimate of Lemma 2.16. We will see in section 4 , how the loss of these structural properties under the assumption of quasi-neutral approximation, leads, for certain data, to nonexistence results for the reduced current driven model. It is also interesting to remark that these properties are somehow "hidden", if one considers the symmetrized form of $(\mathrm{VD})_{V}$. This symmetrized form is derived from $(\mathrm{VD})_{V}$, by using the unknowns $(\psi, n \exp (-\psi), p \exp (\psi))$ instead of $(\psi, n, p)$. Under this form, the existence results for $(\mathrm{VD})_{V}$ which are given in the literature (see e.g. [11] and the references therein) lead to a priori estimates on the currents $I$, which are of the form $c_{1} \exp (-|V|) \leq|I| \leq c_{2} \exp (|V|)$. Of course, such estimates do not lead to existence results for $(\mathrm{CD})_{I}$, contrary to the estimates given in Lemmas 2.9 and 2.16.

We have not analyzed in this paper the impact of the physical scales on certain mathematical properties of the drift-diffusion models. It is well known for instance that the smallness of $\varepsilon$ allows to analyze the drift-diffusion model in the framework of singular perturbation theory (see e.g. [5]). Many other studies in the literature analyze the play of certain physical scales on the drift-diffusion model. As we already mentioned in the introduction, our purpose in this paper is to show that the drift-diffusion models possess general and formal mathematical properties (given in the above and following theorems) which depend only on the structure of the equations and not on the physical scales.

Remark. Theorem 2.7, and, in particular, Lemmas 2.9 and 2.16, show that the currents corresponding to the (possibly multiple) solutions of $(\mathrm{VD})_{V}$ never saturate (i.e. reach a finite limit) as $V$ goes to $\pm \infty$.

We deduce from Theorems 2.1 and 2.7 the following corollary:

Corollary 2.17. Assume that $N$ is in $L^{\infty}(\Omega)$ and that $\mu_{n}$ and $\mu_{p}$ are strictly positive constants. Then for every $I$ in $\mathbb{R},(\mathrm{CD})_{I}$ has a unique weak solution $\left(\psi, n, p, J_{n}\right)$.

\section{Qualitative Properties of the solutions of the Voltage AND CURRENT DRIVEN MODELS}

This section is organized as follows. We first give in Theorem 3.1, the general qualitative properties of the voltage current characteristic. Then we describe the asymptotic behavior of the voltage current characteristic in Lemmas 3.2, 3.9 and Corollary 3.10. The proof of Lemma 3.2 requires intermediate results which are given in Propositions 3.3-3.8. At the end of the section, we give in Lemmas 3.11 and 3.12 , the monotonicity properties mentioned in the introduction.

We introduce the following spaces equipped with their usual norms

$$
E=H^{2}(\Omega) \times\left(H^{1}(\Omega)\right)^{2} \times \mathbb{R}, \quad F=\left(L^{2}(\Omega)\right)^{3} \times \mathbb{R}^{5},
$$

and we set $U=\left(\psi, n, p, J_{n}\right)$. Then, for each fixed $I$ in $\mathbb{R},(\mathrm{CD})_{I}$ can be written under the abstract form

$$
\mathcal{G}(I, U)=0
$$


where $\mathcal{G}$ is the map defined from $\mathbb{R} \times E$ to $F$ by

$$
\begin{aligned}
& \mathcal{G}(I, U)=\left(\varepsilon \psi^{\prime \prime}-(n-p-N), \mu_{n}\left(n^{\prime}-n \psi^{\prime}\right)-J_{n},\right. \\
& \mu_{p}\left(p^{\prime}+p \psi^{\prime}\right)-\left(J_{n}-I\right), \psi(-1)-\psi_{-1}, \\
& \left.n(-1)-n_{-1}, n(1)-n_{1}, p(-1)-p_{-1}, p(1)-p_{1}\right) .
\end{aligned}
$$

From Corollary 2.17, we know that for every $I$, (3.1) admits a unique solution $U$ in $E$. We have the following results

Theorem 3.1. Assume that the hypotheses of Corollary 2.17 hold, and denote by $U$ the map

$$
I \in \mathbb{R} \mapsto U(I) \in E,
$$

where $U(I)$ is the unique solution of (3.1). Then the following properties hold

(i) $U$ is an analytic function and the voltage function $\mathcal{V}$

$$
I \mapsto V
$$

is well defined, onto, analytic on $\mathbb{R}$, and it satisfies

$$
\mathcal{V}(I) \mapsto \pm \infty \quad \text { if and only if } \quad I \mapsto \pm \infty .
$$

(ii) If the data $N, \mu_{n}, \mu_{p}, \varepsilon$ and $\delta$ are such that there exists $V_{c}^{+}$(resp. $V_{c}^{-}$) such that all the solutions of $(\mathrm{VD})_{V}$ are isolated for every $V \geq V_{c}^{+}\left(\right.$resp. $\left.V \leq V_{c}^{-}\right)$, then $(\mathrm{VD})_{V}$ has a unique solution for every $V \geq V_{c}^{+}$(resp. $V \leq V_{c}^{-}$) and the current $I$ is a strictly increasing function of $V$ on $\left[V_{c}^{+},+\infty\right)$ (resp. $\left.\left(-\infty, V_{c}^{-}\right]\right)$.

Proof. It is easy to check that $\mathcal{G}$ is a analytic map from $\mathbb{R} \times E$ to $F$. Now, by using a proof similar to that of Theorem 2.1, we show that for every $I$ in $\mathbb{R}$, the linearized operator $\partial_{U} \mathcal{G}(I, U(I))$ is one-to-one. Since this operator is a Fredholm operator of index 0 , we can apply the implicit function theorem. This together with the global uniqueness Theorem 2.17 imply that $U$ is continuously Fréchet differentiable from $\mathbb{R}$ to $E$. Thanks to the fact that $\mathcal{G}$ is analytic, we deduce that $U$ is also analytic by applying the analytic version of the implicit function theorem. Since (3.1) has a unique solution $\left(\psi, n, p, J_{n}\right)$ for every $I$, the voltage $V$ is uniquely defined by the relation (1.10), which proves that the voltage function $\mathcal{V}$ is well defined. From (1.10) and since $U$ is analytic, we deduce that $\mathcal{V}$ is analytic.

From Lemmas 2.9 and 2.16, we already know that if $\mathcal{V}(I)$ goes to $\pm \infty$ then $I$ goes to $\pm \infty$. On the other hand, the projection of $\mathcal{B}$ on the $V$-axis equals $\mathbb{R}$. This, together with the continuity of $\mathcal{V}$ imply that $\mathcal{V}(I)$ goes to $\pm \infty$ if $I$ goes to $\pm \infty$ and that $\mathcal{V}$ is onto.

Let us now assume that the data $N, \mu_{n}, \mu_{p}, \varepsilon$ and $\delta$ are such that all the solutions of $(\mathrm{VD})_{V}$ are isolated for all $V \geq V_{c}^{+}$. We now prove that $(\mathrm{VD})_{V}$ has a unique weak solution for all $V \geq V_{c}^{+}$. Assume to the contrary that there exists $V$ in $\left[V_{c}^{+},+\infty\right)$, such that $(\mathrm{VD})_{V}$ has at least two different solutions $\left(\psi, n, p, J_{n}, I\right)$ and $\left(\tilde{\psi}, \tilde{n}, \tilde{p}, \tilde{J}_{n}, \tilde{I}\right)$. From Corollary 2.17, we deduce that we have $I \neq \tilde{I}$. Moreover we can assume without loss of generality that $I<\tilde{I}$ holds. Hence, we have

$$
\mathcal{V}(I)=\mathcal{V}(\tilde{I})=V .
$$

Since $\mathcal{V}$ is continuous on $\mathbb{R}, \mathcal{V}$ attains at least one extremum at a point of the interval $(I, \tilde{I})$. If $I^{\star}$ is a point of $(I, \tilde{I})$ at which $\mathcal{V}$ attains an extremum, we denote by $\left(\psi^{\star}, n^{\star}, p^{\star}, J_{n}^{\star}\right)$ the unique solution of $(\mathrm{CD})_{I^{\star}}$. From the implicit function theorem and since $\mathcal{V}$ is continuous on $\mathbb{R}$, we deduce that $\left(\psi^{\star}, n^{\star}, p^{\star}, J_{n}^{\star}, I^{\star}\right)$ is a non-isolated solution of $(\mathrm{VD})_{V^{\star}}$, where $V^{\star}=\mathcal{V}\left(I^{\star}\right)$. On the other hand, from our hypothesis, 
$\mathcal{V}$ cannot attain an extremum greater than $V_{c}^{+}$. Hence, if $\mathcal{V}$ attains an extremum at a point $I^{\star}$ of $(I, \tilde{I})$, then we have $\mathcal{V}\left(I^{\star}\right)<V_{c}^{+}$.

We claim that

$$
\mathcal{V}([I, \tilde{I}]) \subset(-\infty, V] .
$$

To the contrary, assume that there exists $\bar{I}$ in $[I, \tilde{I}]$, such that the inequality $V<\mathcal{V}(\bar{I})$ holds. This implies that there exists $I^{\star}$ in $[I, \tilde{I}]$, such that $\mathcal{V}\left(I^{\star}\right)$ is the maximum of $\mathcal{V}$ on the interval $[I, \tilde{I}]$. Hence we have $\mathcal{V}\left(I^{\star}\right)<V_{c}^{+}$. But this is impossible, since we also have

$$
\mathcal{V}\left(I^{\star}\right) \geq \mathcal{V}(\bar{I})>V \geq V_{c}^{+} .
$$

Therefore we proved that $\mathcal{V}([I, \tilde{I}]) \subset(-\infty, V]$. Two cases can now occur: either $\mathcal{V}(-\infty, I] \subset(-\infty, V]$ or not.

Assume first that

$$
\mathcal{V}(-\infty, I] \subset(-\infty, V]
$$

This together with

$$
\mathcal{V}([I, \tilde{I}]) \subset(-\infty, V],
$$

imply that $\mathcal{V}$ attains a maximum at the point $I$, which contradicts the hypothesis. Hence there exists $\bar{I}$ in $(-\infty, I)$ such that $V<\mathcal{V}(\bar{I})$. This is impossible since we have

$$
\lim _{I \rightarrow-\infty} \mathcal{V}(I)=-\infty,
$$

and since $\mathcal{V}$ cannot attain a maximum greater than $V_{c}^{+}$. Hence $(\mathrm{VD})_{V}$ has a unique weak solution for all $V \geq V_{c}^{+}$and moreover $\mathcal{V}$ is monotonic on $\left[\mathcal{V}\left(V_{c}^{+}\right),+\infty\right)$. From Lemma 2.9, we deduce that $\mathcal{V}$ is strictly increasing on $\left[\mathcal{V}\left(V_{c}^{+}\right),+\infty\right)$. The proof is similar if we assume that all the solutions of $(\mathrm{VD})_{V}$ are isolated for all $V \leq V_{c}^{-}$. This concludes the proof of the theorem.

Remark. From Theorem 3.1, we know that the voltage function is single valued and analytic, but this function is not always one-to-one. The inverse function of $\mathcal{V}$ is the (possibly multiple valued) voltage current characteristic. Hence, if $(V, I)$ is a bifurcation point of the voltage current characteristic, then $\mathcal{V}$ attains an extremum at the point $I$. Conversely, if $\mathcal{V}$ attains an extremum at a point $I$, then $(\mathcal{V}(I), I)$ is a bifurcation point of the voltage current characteristic. Moreover, Theorem 3.1 proves that the currents $I$ corresponding to (possible) turning points of the voltage current characteristic are characterized by

$$
\mathcal{N}\left(\partial_{U} \mathcal{G}(I, U(I))\right) \neq\{0\}, \quad \mathcal{V}^{\prime}(I)=0,
$$

where $\mathcal{N}\left(\partial_{U} \mathcal{G}(I, U(I))\right.$ stands for the null space of $\partial_{U} \mathcal{G}(I, U(I))$.

From Lemmas 2.9 and 2.16, we know that all the solutions $\left(\psi, n, p, J_{n}, I\right)$ of $(\mathrm{VD})_{V}$ are such that $|I|$ grows as least as an affine function of $|V|$ as $V$ goes to $+\infty$. The purpose of the next two lemmas is to characterize more precisely the asymptotic behavior of $I$ as $V$ goes to $\pm \infty$.

Lemma 3.2. Assume that the hypotheses of Theorem 2.7 hold and let $V_{2}^{+}$be defined by (2.62). Then there exist two constants $a_{1}^{+}>0$ and $a_{2}^{+}$such that for all $V \geq V_{2}^{+}$, every solution $\left(\psi, n, p, J_{n}, I\right)$ of $(\mathrm{VD})_{V}$ satisfies

$$
a_{1}^{+} V+a_{2}^{+} \geq I \text {. }
$$


We use, as for the proof of Lemma 2.9 some notation which simplify our presentation.

If $\left(\psi, n, p, J_{n}, I\right)$ is a solution of (VD) ${ }_{V}$ such that case (i) (resp. (ii)) of Lemma 2.8 holds, we define a number $X_{e}^{+}$and a function $s_{+}$by setting

$$
X_{e}^{+}=-1,\left(\operatorname{resp} . X_{e}^{+}=1\right),
$$

and,

$$
s_{+}(x)=p(x)\left(\text { resp. } s_{+}(x)=-n(x)\right) \quad \forall x \in \bar{\Omega} .
$$

For each case (i) or (ii) of Lemma 2.8, two subcases can occur Either one of the following two inequalities holds:

$$
(n-p)^{\prime}\left(X_{e}^{+}\right) \leq 0 \quad \text { or } \quad 0 \leq s_{+}^{\prime}\left(X_{e}^{+}\right),
$$

or the following two inequalities hold:

$$
0<(n-p)^{\prime}\left(X_{e}^{+}\right) \quad \text { and } \quad s_{+}^{\prime}\left(X_{e}^{+}\right)<0 .
$$

The case where (3.6) (resp. (3.7)) holds is treated in Proposition 3.4 (resp. Propositions $3.6,3.7,3.8)$.

If $\left(\psi, n, p, J_{n}, I\right)$ is a solution of $(\mathrm{VD})_{V}$ such that (3.7) holds, then since $(n-p)$ is in $\mathcal{C}^{1}(\bar{\Omega})$, this solution satisfies either

$$
(n-p)^{\prime} \geq 0 \quad \text { on } \bar{\Omega},
$$

or,

$$
\exists x \in \Omega \quad \text { such that } \quad 0>(n-p)^{\prime}(x) .
$$

In both cases (3.8) or (3.9), there exists $X_{m}^{+}$in $\bar{\Omega}$ such that the following properties hold:

$$
X_{m}^{+} \neq X_{e}^{+}
$$

and,

$$
(n-p)^{\prime}(x) \geq 0 \quad \forall x \in\left[\min \left(X_{m}^{+}, X_{e}^{+}\right), \max \left(X_{m}^{+}, X_{e}^{+}\right)\right] .
$$

Moreover, if (3.8) (resp. (3.9) holds, we can choose $X_{m}^{+}$such that

$$
X_{m}^{+}=-X_{e}^{+} \quad\left(\text { resp. }(n-p)^{\prime}\left(X_{m}^{+}\right)=0\right) .
$$

Hence if (3.7) holds, it is always possible to choose $X_{m}^{+}$such that (3.10), (3.11) and (3.12) hold.

The proof of Lemma 3.2 is based on the next Propositions 3.3, 3.4, 3.5, 3.6, 3.7, and 3.8 .

Proposition 3.3. Assume that the hypotheses of Theorem 2.7 hold and let $V \geq 0$ be given. Then any solution $\left(\psi, n, p, J_{n}, I\right)$ of $(\mathrm{VD})_{V}$ satisfies

$$
\psi^{\prime}\left(X_{e}^{+}\right) \geq 2^{-1}\left[U_{b i}-V-\frac{2\left(N_{+}-N_{-}\right)}{\varepsilon}\right],
$$

and,

$$
\psi^{\prime}\left(-X_{e}^{+}\right) \leq 2^{-1}\left[U_{b i}-V+\frac{2\left(N_{+}-N_{-}\right)}{\varepsilon}\right],
$$


where $U_{b i}$ and $X_{e}^{+}$are respectively defined in (2.45) and (3.4). Moreover, if the case (i) (resp. (ii)) of Lemma 2.8 holds, then any solution $\left(\psi, n, p, J_{n}, I\right)$ of $(\mathrm{VD})_{V}$ satisfies

$$
\begin{gathered}
\frac{I \mu_{p}}{\mu_{n}+\mu_{p}} \leq I-J_{n}, \\
\left(\text { resp. } \quad \frac{I \mu_{n}}{\mu_{n}+\mu_{p}} \leq J_{n}\right) .
\end{gathered}
$$

Proof. Let $V \geq 0$ be given and let $\left(\psi, n, p, J_{n}, I\right)$ be an arbitrary solution of $(\mathrm{VD})_{V}$. We define $I_{V}$ and $J_{V}$ as in (2.23). Since $V \geq 0$ holds, we deduce from (2.43) that we have

$$
I_{V}=0 \quad \Longleftrightarrow \quad J_{V}=0 .
$$

Assume first that (i) holds. Therefore from Lemma 2.8 we deduce that (2.24) is satisfied. From (2.24) and (1.1) we have

$$
\psi^{\prime \prime}(t) \leq\left(N_{+}-N_{-}\right) \varepsilon^{-1} \quad \text { for a.e. } t \in \Omega .
$$

Double integration of this inequality from $t=-1$ to $t=x$, and then from $x=-1$ to $x=1$ gives (3.13) with $X_{e}^{+}=-1$. Moreover, since (i), (3.17) and the last inequality of (2.43) hold, we deduce that we have $J_{V} \leq 0$, which in turn implies (3.15).

Assume now that (ii) holds, so that from Lemma 2.8, (2.25) is satisfied. From (2.25) and (1.1), we deduce that

$$
\psi^{\prime \prime}(t) \geq-\left(N_{+}-N_{-}\right) \varepsilon^{-1} \quad \text { for a.e. } t \in \Omega \text {. }
$$

Double integration of this inequality from $t=x$ to $t=1$, and then from $x=-1$ to $x=1$ gives (3.13) with $X_{e}^{+}=1$. Moreover, since (ii), (3.17) and the last inequality of (2.43) hold, we deduce that we have $J_{V} \geq 0$, which in turn yields to (3.16).

In order to prove (3.14), we remark that $X_{e}^{+}=-x_{e}^{+}$, where $x_{e}^{+}$is defined by (2.34). Hence the inequality (2.44) proved in Proposition 2.10 implies (3.14). This concludes the proof of the proposition.

Proposition 3.4. Assume that the hypotheses of Theorem 2.7 hold. Then there exists $W_{1}^{+} \geq 0$ defined by (3.36) such that, for all $V \geq W_{1}^{+}$and for every solution $\left(\psi, n, p, J_{n}, I\right)$ of $(\mathrm{VD})_{V}$ which satisfies (3.6), the following estimate holds:

$$
I \leq \mu_{+} k_{+}\left[V-\left(U_{b i}-2\left(N_{+}-N_{-}\right) / \varepsilon\right)\right],
$$

where $U_{b i}$ is defined by (2.45) and where $\mu_{+}$and $k_{+}$are respectively given by

$$
\begin{gathered}
\mu_{+}=\max \left(\mu_{n}, \mu_{p}\right)>0, \\
k_{+}=\max \left(n_{1}+p_{1}, n_{-1}+p_{-1}\right)>0 .
\end{gathered}
$$

Proof. Let $V \geq 0$ be given and let $\left(\psi, n, p, J_{n}, I\right)$ be a solution of $(\mathrm{VD})_{V}$ such that (3.6) holds.

Let us first assume that the first inequality of (3.6) holds. From (1.2) and (1.3), we recall that we have

$$
(n-p)^{\prime}=(n+p) \psi^{\prime}+J_{n} \mu_{n}^{-1}+\left(I-J_{n}\right) \mu_{p}^{-1} .
$$


Setting $x=X_{e}^{+}$in this last equation and using (3.13) and the fact that $(n+p)\left(X_{e}^{+}\right)>0$, we get

$$
J_{n} \mu_{n}^{-1}+\left(I-J_{n}\right) \mu_{p}^{-1} \leq 2^{-1}(n+p)\left(X_{e}^{+}\right)\left[V-\left(U_{b i}-2\left(N_{+}-N_{-}\right) \varepsilon^{-1}\right)\right] .
$$

From (2.43), we deduce that for $V \geq W_{1}^{+}$(3.18) holds.

Assume now that the second inequality of (3.6) holds. We have to consider the cases (i) and (ii) of Lemma 2.8 separately.

Assume first that (i) holds, so that from Proposition 3.3, we have (3.15). Since (i) holds we have from (3.4), $X_{e}^{+}=-1$ and $s_{+}=p$. Moreover, we have assumed that $s_{+}^{\prime}\left(X_{e}^{+}\right) \geq 0$ hold. Hence we have from (1.3)

$$
I-J_{n} \leq-\mu_{p} p\left(X_{e}^{+}\right) \psi^{\prime}\left(X_{e}^{+}\right)
$$

From (3.13), we deduce that

$$
I-J_{n} \leq 2^{-1} \mu_{p} p_{-1}\left[V-\left(U_{b i}-2\left(N_{+}-N_{-}\right) \varepsilon^{-1}\right)\right]
$$

holds. This together with (3.15) imply that (3.18) holds for $V \geq W_{1}^{+}$.

Assume now that (ii) holds, so that from Proposition 3.3, we have (3.16). Since (ii) holds we have from (3.4), $X_{e}^{+}=1$ and $s_{+}=-n$. Moreover, we have assumed that $s_{+}^{\prime}\left(X_{e}^{+}\right) \geq 0$ hold. Hence we have from (1.2)

$$
J_{n} \leq-\mu_{n} n\left(X_{e}^{+}\right) \psi^{\prime}\left(X_{e}^{+}\right)
$$

From (3.13), we deduce that

$$
J_{n} \leq 2^{-1} \mu_{n} n_{1}\left[V-\left(U_{b i}-2\left(N_{+}-N_{-}\right) \varepsilon^{-1}\right)\right]
$$

holds. This together with (3.16) imply that (3.18) holds for $V \geq W_{1}^{+}$. This concludes the proof of the proposition.

Proposition 3.5. Assume that the hypotheses of Theorem 2.7 hold. Then for all $V \geq 0$ and for every solution $\left(\psi, n, p, J_{n}, I\right)$ of $(\mathrm{VD})_{V}$ satisfying (3.7), the following estimate holds:

$\psi^{\prime}(x) \geq 2^{-1}\left(U_{b i}-6\left(N_{+}-N_{-}\right) / \varepsilon-V\right) \quad \forall x \in\left[\min \left(X_{m}^{+}, X_{e}^{+}\right), \max \left(X_{m}^{+}, X_{e}^{+}\right)\right]$,

where $X_{e}^{+}$is defined as in (3.4) and where $X_{m}^{+}$is chosen such that (3.10), (3.11) and (3.12) hold.

Proof. Let $V \geq 0$ be given and let $\left(\psi, n, p, J_{n}, I\right)$ be a solution of $(\mathrm{VD})_{V}$ such that (3.7) holds. By our choice of $X_{m}^{+},(3.11)$ holds. Therefore we have for all $x$ in $\left[\min \left(X_{m}^{+}, X_{e}^{+}\right), \max \left(X_{m}^{+}, X_{e}^{+}\right)\right]$

$$
(n-p)\left(\max \left(X_{e}^{+}, X_{m}^{+}\right)\right) \geq(n-p)(x) \geq(n-p)\left(\min \left(X_{e}^{+}, X_{m}^{+}\right)\right) .
$$

Assume first that $X_{e}^{+}=-1$. Then we get from the left hand side of (3.22) and from (1.1)

$$
\psi^{\prime \prime}(x) \geq-\left(N_{+}-N_{-}\right) \varepsilon^{-1} \quad \text { for a.e. } x \in\left[-1, X_{m}^{+}\right] .
$$

For $x$ in $\left[-1, X_{m}^{+}\right]$, we integrate this inequality from -1 to $x$. This together with (3.13) give (3.21). We get (3.21) in the same way for the case $X_{e}^{+}=1$. This concludes the proof of the proposition. 
Proposition 3.6. Assume that the hypotheses of Theorem 2.7 hold and let $W_{2}^{+} \geq 0$ be defined by (3.37). Assume moreover that $\left(\psi, n, p, J_{n}, I\right)$ is a solution of $(\mathrm{VD})_{V}$ with $V \geq W_{2}^{+}$, such that (3.7) holds. We define $X_{e}^{+}$and the function $s_{+}$respectively by (3.4) and (3.5), whereas $X_{m}^{+}$is chosen as in Proposition 3.5. Then if $s_{+}^{\prime}$ changes sign in $\left(\min \left(X_{m}^{+}, X_{e}^{+}\right), \max \left(X_{m}^{+}, X_{e}^{+}\right)\right)$, the following estimate holds:

$$
I \leq \mu_{+} \max \left(n_{1}, p_{-1}\right)\left[V-\left(U_{b i}-6\left(N_{+}-N_{-}\right) / \varepsilon\right)\right],
$$

where $\mu_{+}$is defined by (3.19).

Proof. Let $V \geq W_{2}^{+}$be given and let $\left(\psi, n, p, J_{n}, I\right)$ be a solution of $(\mathrm{VD})_{V}$ such that (3.7) holds and such that $s_{+}^{\prime}$ changes sign in $\left(\min \left(X_{m}^{+}, X_{e}^{+}\right), \max \left(X_{m}^{+}, X_{e}^{+}\right)\right)$.

Assume first that the case (i) of Lemma 2.8 holds, so that from (3.4) and (3.5) we have $X_{e}^{+}=-1$ and $s_{+}=p$. Moreover from Proposition 3.3 and since $(i)$ holds, (3.15) is satisfied. Since (3.7) holds, we have $p^{\prime}(-1)<0$. By hypothesis, $p^{\prime}$ changes sign in $\left(-1, X_{m}^{+}\right)$, therefore there exists $Y_{m}^{+}$in $\left(-1, X_{m}^{+}\right)$such that

$$
p^{\prime} \leq 0 \quad \text { on }\left[-1, Y_{m}^{+}\right], \quad \text { and } \quad p^{\prime}\left(Y_{m}^{+}\right)=0 .
$$

From (1.3) and the equality $p^{\prime}\left(Y_{m}^{+}\right)=0$, we deduce that

$$
I-J_{n}=-\mu_{p} p\left(Y_{m}^{+}\right) \psi^{\prime}\left(Y_{m}^{+}\right) \text {. }
$$

Now since $Y_{m}^{+}$is in $\left(-1, X_{m}^{+}\right)$, we can set $x=Y_{m}^{+}$in (3.21). The resulting inequality, together with the inequality $\mu_{p} p\left(Y_{m}^{+}\right)>0$ imply

$$
I-J_{n} \leq 2^{-1} \mu_{p} p\left(Y_{m}^{+}\right)\left[V-\left(U_{b i}-6\left(N_{+}-N_{-}\right) \varepsilon^{-1}\right)\right] .
$$

From (3.15) and the above inequality, we get

$$
I \leq 2^{-1}\left(\mu_{n}+\mu_{p}\right) p\left(Y_{m}^{+}\right)\left[V-\left(U_{b i}-6\left(N_{+}-N_{-}\right) \varepsilon^{-1}\right)\right] .
$$

On the other hand, since we have $p^{\prime} \leq 0$ on $\left[-1, Y_{m}^{+}\right]$, we get $p_{-1} \geq p\left(Y_{m}^{+}\right)$. This, together with (3.24) and the inequality $V \geq W_{2}^{+}$leads to (3.23).

Assume now that (ii) holds, so that from (3.4) and (3.5) we have $X_{e}^{+}=1$ and $s_{+}=-n$. Moreover from Proposition 3.3 and since (ii) holds, (3.16) is satisfied. Since by hypothesis (3.7) holds, we have $n^{\prime}(1)>0$. By hypothesis, $n^{\prime}$ changes sign in $\left(X_{m}^{+}, 1\right)$, therefore there exists $Z_{m}^{+}$in $\left(X_{m}^{+}, 1\right)$ such that

$$
n^{\prime} \geq 0 \quad \text { on }\left[Z_{m}^{+}, 1\right], \quad \text { and } n^{\prime}\left(Z_{m}^{+}\right)=0 .
$$

From (1.2) and the equality $n^{\prime}\left(Z_{m}^{+}\right)=0$, we deduce that

$$
J_{n}=-\mu_{n} n\left(Z_{m}^{+}\right) \psi^{\prime}\left(Z_{m}^{+}\right) .
$$

Since $Z_{m}^{+}$is in $\left(X_{m}^{+}, 1\right)$, we can set $x=Z_{m}^{+}$in (3.21). The resulting inequality, together with the inequality $\mu_{n} n\left(Z_{m}^{+}\right)>0$ implies

$$
J_{n} \leq 2^{-1} \mu_{n} n\left(Z_{m}^{+}\right)\left[V-\left(U_{b i}-6\left(N_{+}-N_{-}\right) \varepsilon^{-1}\right)\right] .
$$

On the other hand, since we have $n^{\prime} \geq 0$ on $\left[Z_{m}^{+}, 1\right]$, we have $n_{1} \geq n\left(Z_{m}^{+}\right)$. This, together with (3.16) and the inequality $W \geq V_{2}^{+}$lead to (3.23). This concludes the proof of the proposition.

The only case which remains now is the case where (3.7) holds and where $s_{+}^{\prime}$ keeps a constant sign in $\left[\min \left(X_{m}^{+}, X_{e}^{+}\right), \max \left(X_{m}^{+}, X_{e}^{+}\right)\right]$. For this last case, we have to consider the two subcases given respectively by (3.8) and (3.9). These two subcases are treated respectively in the next two propositions. 
Proposition 3.7. Assume that the hypotheses of Theorem 2.7 hold and let $V_{2}^{+}$be defined by (2.62). For $V \geq V_{2}^{+}$, let $\left(\psi, n, p, J_{n}, I\right)$ be a solution of $(\mathrm{VD})_{V}$, such that (3.7) holds and such that $s_{+}^{\prime}$ keeps a constant sign in $\left[\min \left(X_{m}^{+}, X_{e}^{+}\right), \max \left(X_{m}^{+}, X_{e}^{+}\right)\right]$. Then if (3.8) holds, the following estimate is satisfied:

$$
I \leq 2^{-1} \mu_{+}\left[\max \left(2 n_{1}-N_{-}, 2 p_{-1}+N_{+}\right)\left(V-U_{b i}\right)+\left(N_{+}-N_{-}\right)\right],
$$

where $\mu_{+}$is defined by (3.19).

Proof. Let $V \geq V_{2}^{+}$be given and assume that $\left(\psi, n, p, J_{n}, I\right)$ is a solution of (VD) $V$ such that (3.7) holds and such that $s_{+}^{\prime}$ keeps a constant sign in $\left[\min \left(X_{m}^{+}, X_{e}^{+}\right)\right.$, $\left.\max \left(X_{m}^{+}, X_{e}^{+}\right)\right]$. If (3.8) holds, we have from (3.12): $X_{m}^{+}=-X_{e}^{+}$. Since $X_{e}^{+}$is in $\{-1,1\}$, we get

$$
\min \left(X_{m}^{+}, X_{e}^{+}\right)=-1, \max \left(X_{m}^{+}, X_{e}^{+}\right)=1
$$

Assume first that the case (i) of Lemma 2.8 holds, so that we have $X_{e}^{+}=-1$ and $s_{+}=p$. Since (3.7) holds and since $s_{+}^{\prime}$ keeps a constant sign in $[-1,1]$, we have

$$
p_{-1} \geq p(x) \quad \forall x \in[-1,1] .
$$

On the other hand, since (i) holds, we deduce from Lemma 2.8 that (2.24) holds. Hence we have

$$
n(x) \leq p(x)+N_{+} \quad \forall x \in[-1,1] .
$$

This, together with (3.26) yield to

$$
(n+p)(x) \leq 2 p_{-1}+N_{+} \quad \forall x \in[-1,1] .
$$

Assume now that the case (ii) of Lemma 2.8 holds, so that we have $X_{e}^{+}=1$ and $s_{+}=-n$. Since $(3.7)$ holds and since $s_{+}^{\prime}$ keeps a constant sign in $[-1,1]$, we have

$$
n(x) \leq n_{1} \quad \forall x \in[-1,1] .
$$

On the other hand, since (ii) holds, we deduce from Lemma 2.8 that (2.25) holds. Hence we have

$$
p(x) \leq n(x)-N_{-} \quad \forall x \in[-1,1] .
$$

This, together with (3.28) yield

$$
(n+p)(x) \leq 2 n_{1}-N_{-} \quad \forall x \in[-1,1] .
$$

From (3.27) and (3.29), we deduce that for both cases (i) and (ii) of Lemma 2.8, the following inequality holds:

$$
(n+p)(x) \leq \max \left(2 n_{1}-N_{-}, 2 p_{-1}+N_{+}\right) \quad \forall x \in[-1,1] .
$$

Now from (1.1) and (3.8), we have

$$
\text { - }\left(N_{+}-N_{-}\right) \varepsilon^{-1} \leq \psi^{\prime \prime}(x) \leq\left(N_{+}-N_{-}\right) \varepsilon^{-1} \quad \text { for a.e. } x \in \Omega .
$$

On the other hand, from Proposition 3.3, we know that (3.14) holds. The inequality (3.14), together with (3.31) imply that for $V \geq V_{2}^{+}$, the following inequality holds:

$$
\psi^{\prime}(x) \leq 0 \quad \forall x \in[-1,1] .
$$

From (1.2) and (1.3), we get

$$
(n-p)^{\prime}(x)=(n+p)(x) \psi^{\prime}(x)+J_{n} \mu_{n}^{-1}+\left(I-J_{n}\right) \mu_{p}^{-1} \quad \forall x \in[-1,1] .
$$


Using (3.30) and (3.32) in this last equality and integrating the resulting inequality from $x=-1$ to $x=1$, we obtain

$J_{n} \mu_{n}^{-1}+\left(I-J_{n}\right) \mu_{p}^{-1} \leq 2^{-1}\left[\left(N_{+}-N_{-}\right)+\max \left(2 n_{1}-N_{-}, 2 p_{-1}+N_{+}\right)\left(V-U_{b i}\right)\right]$. which in turn implies (3.25). This concludes the proof of the proposition.

We now consider the case where (3.9) holds.

Proposition 3.8. Assume that the hypotheses of Theorem 2.7 hold and let $W_{2}^{+}$be defined by (3.37). For $V \geq W_{2}^{+}$, let $\left(\psi, n, p, J_{n}, I\right)$ be a solution of $(\mathrm{VD})_{V}$, such that (3.7) holds and such that $s_{+}^{\prime}$ keeps a constant sign in $\left[\min \left(X_{m}^{+}, X_{e}^{+}\right), \max \left(X_{m}^{+}, X_{e}^{+}\right)\right]$. Then if (3.9) holds, the following estimate is satisfied:

$$
I \leq 2^{-1} \mu_{+} \max \left(2 n_{1}-N_{-}, 2 p_{-1}+N_{+}\right)\left[V-\left(U_{b i}-6\left(N_{+}-N_{-}\right) / \varepsilon\right)\right],
$$

where $\mu_{+}$is defined by (3.19).

Proof. Let $V \geq W_{2}^{+}$be given and assume that $\left(\psi, n, p, J_{n}, I\right)$ is a solution of $(\mathrm{VD})_{V}$ such that (3.7) holds and such that $s_{+}^{\prime}$ keeps a constant sign in $\left[\min \left(X_{m}^{+}, X_{e}^{+}\right)\right.$, $\left.\max \left(X_{m}^{+}, X_{e}^{+}\right)\right]$. From this, we deduce as in the proof of Proposition 3.7 that

$$
(n+p)\left(X_{m}^{+}\right) \leq \max \left(2 n_{1}-N_{-}, 2 p_{-1}+N_{+}\right) .
$$

Since (3.9) is assumed to hold, we have from (3.12)

$$
(n-p)^{\prime}\left(X_{m}^{+}\right)=0 \text {. }
$$

Setting $x=X_{m}^{+}$in (1.2) and (1.3) and using the above equality, we get

$$
J_{n} \mu_{n}^{-1}+\left(I-J_{n}\right) \mu_{p}^{-1}=-(n+p)\left(X_{m}^{+}\right) \psi^{\prime}\left(X_{m}^{+}\right) .
$$

Setting $x=X_{m}^{+}$in (3.21) and using the resulting inequality in the above equality, we obtain

$$
J_{n} \mu_{n}^{-1}+\left(I-J_{n}\right) \mu_{p}^{-1} \leq 2^{-1}(n+p)\left(X_{m}^{+}\right)\left[V-\left(U_{b i}-6\left(N_{+}-N_{-}\right) \varepsilon^{-1}\right)\right] .
$$

The above inequality together with (3.35) imply that (3.34) holds for $V \geq W_{2}^{+}$. This concludes the proof of the proposition.

We are now able to prove Lemma 3.2

Proof of Lemma 3.2. We define $V_{2}^{+}$by $(2.62)$ and $W_{1}^{+}$and $W_{2}^{+}$respectively by

$$
W_{1}^{+}=\max \left(0, U_{b i}-2\left(N_{+}-N_{-}\right) / \varepsilon\right) \text {, }
$$

and,

$$
W_{2}^{+}=\max \left(0, U_{b i}-6\left(N_{+}-N_{-}\right) / \varepsilon\right) .
$$

Let $V \geq V_{2}^{+}$be given and let $\left(\psi, n, p, J_{n}, I\right)$ be a solution of $(\mathrm{VD})_{V}$. Then this solution satisfies either (3.6) or (3.7). Assume first that this solution satisfies (3.6). Since $V_{2}^{+} \geq W_{1}^{+}$, we deduce from Proposition 3.4 that (3.18) holds. Assume now that (3.7) holds. Let $X_{e}^{+}, s_{+}$, and $X_{m}^{+}$be defined as above. Then either $s_{+}^{\prime}$ changes sign in $\left(\min \left(X_{m}^{+}, X_{e}^{+}\right), \max \left(X_{m}^{+}, X_{e}^{+}\right)\right)$or it keeps a constant sign in this interval. If $s_{+}^{\prime}$ changes sign in $\left(\min \left(X_{m}^{+}, X_{e}^{+}\right), \max \left(X_{m}^{+}, X_{e}^{+}\right)\right)$, we deduce from Proposition 3.6 and since $V \geq W_{2}^{+}$holds, that (3.23) is satisfied. If $s_{+}^{\prime}$ keeps a constant sign in $\left[\min \left(X_{m}^{+}, X_{e}^{+}\right), \max \left(X_{m}^{+}, X_{e}^{+}\right)\right]$, two subcases can occur: either (3.8) or (3.9) holds. If (3.8) holds, we use Proposition 3.7. We deduce that since $V \geq V_{2}^{+}$holds, (3.25) 
is satisfied. If (3.9) holds, we use Proposition 3.8. We deduce that since $V \geq V_{2}^{+}$ holds, (3.34) is satisfied.

From all these cases we deduce that (3.3) holds with $V_{2}^{+}$given by (2.62), and $a_{1}^{+}>0$ and $a_{2}^{+}$given respectively by

$$
a_{1}^{+}=\mu_{+} \max \left(n_{1}+p_{1}, n_{-1}+p_{-1}, p_{-1}+2^{-1} N_{+}, n_{1}-2^{-1} N_{-}\right),
$$

and,

$$
a_{2}^{+}=-a_{1}^{+} U_{b i}+\left(N_{+}-N_{-}\right) \varepsilon^{-1} \max \left(2^{-1} \mu_{+} \varepsilon, 6 a_{1}^{+}\right) .
$$

This concludes the proof of the lemma.

We now consider the case where $V \leq 0$ holds. We obtain in this case the following lemma, which is analogous to Lemma 3.2.

Lemma 3.9. Assume that the hypotheses of Theorem 2.7 hold and let $V_{2}^{-} \leq 0$ be defined by (2.76). Then there exist two constants $a_{1}^{-}>0$ and $a_{2}^{-}$such that for all $V \leq V_{2}^{-}$, every solution $\left(\psi, n, p, J_{n}, I\right)$ of $(\mathrm{VD})_{V}$ satisfies

$$
a_{1}^{-} V+a_{2}^{-} \leq I \text {. }
$$

The proof of this lemma is similar to that of Lemma 3.2, provided that some changes are taken into account. First, we now use the following notation:

If $\left(\psi, n, p, J_{n}, I\right)$ is a solution of $(\mathrm{VD})_{V}$ such that case (i) (resp. (ii)) of Lemma 2.8 holds, we define a number $X_{e}^{-}$and a function $s_{-}$by setting

$$
X_{e}^{-}=1 \quad\left(\text { resp. } X_{e}^{-}=-1\right),
$$

and,

$$
s_{-}(x)=-p(x) \quad\left(\text { resp. } s_{-}(x)=n(x)\right) \quad \forall x \in \bar{\Omega} .
$$

Any solution $\left(\psi, n, p, J_{n}, I\right)$ of $(\mathrm{VD})_{V}$ satisfies either one of the following two inequalities:

$$
0 \leq(n-p)^{\prime}\left(X_{e}^{-}\right) \quad \text { or } \quad 0 \leq s_{-}^{\prime}\left(X_{e}^{-}\right),
$$

or it satisfies the following two inequalities:

$$
(n-p)^{\prime}\left(X_{e}^{-}\right)<0 \quad \text { and } \quad s_{-}^{\prime}\left(X_{e}^{-}\right)<0 .
$$

If $\left(\psi, n, p, J_{n}, I\right)$ is a solution of $(\mathrm{VD})_{V}$ such that (3.44) holds, then this solution satisfies either

$$
(n-p)^{\prime} \leq 0 \quad \text { on } \quad \bar{\Omega},
$$

or,

$$
\exists x \in \Omega \quad \text { such that } \quad 0<(n-p)^{\prime}(x) .
$$

In both cases (3.45) or (3.46), there exists $X_{m}^{-}$in $\bar{\Omega}$ such that the following properties hold:

$$
X_{m}^{-} \neq X_{e}^{-},
$$

and,

$$
(n-p)^{\prime}(x) \leq 0 \quad \forall x \in\left[\min \left(X_{m}^{-}, X_{e}^{-}\right), \max \left(X_{m}^{-}, X_{e}^{-}\right)\right] .
$$

Moreover, if (3.45) (resp. (3.46)) holds, we can choose $X_{m}^{-}$such that

$$
X_{m}^{-}=-X_{e}^{-} \quad\left(\operatorname{resp} .(n-p)^{\prime}\left(X_{m}^{-}\right)=0\right) .
$$


Hence if (3.44) holds, it is always possible to choose $X_{m}^{-}$such that (3.47), (3.48) and (3.49) hold.

Proof of Lemma 3.9. We first remark that if $V \leq 0$ holds, then a proof similar to that of Proposition 3.3 shows that any solution $\left(\psi, n, p, J_{n}, I\right)$ of $(\mathrm{VD})_{V}$ satisfies

$$
\psi^{\prime}\left(X_{e}^{-}\right) \leq 2^{-1}\left(U_{b i}+2\left(N_{+}-N_{-}\right) \varepsilon^{-1}-V\right),
$$

and,

$$
\psi^{\prime}\left(-X_{e}^{-}\right) \geq 2^{-1}\left(U_{b i}-2\left(N_{+}-N_{-}\right) \varepsilon^{-1}-V\right) .
$$

Moreover if the case (i) (resp. (ii)) of Lemma 2.8 holds, then any solution $(\psi, n, p$, $\left.J_{n}, I\right)$ of $(\mathrm{VD})_{V}$ satisfies

$$
\begin{gathered}
\frac{I \mu_{p}}{\mu_{n}+\mu_{p}} \geq I-J_{n}, \\
\text { (resp. } \left.\quad \frac{I \mu_{n}}{\mu_{n}+\mu_{p}} \geq J_{n}\right) .
\end{gathered}
$$

We define $V_{1}^{-}$and $V_{2}^{-}$respectively by (2.75) and (2.76). Let $V \leq V_{2}^{-}$be given and let $\left(\psi, n, p, J_{n}, I\right)$ be a solution of $(\mathrm{VD})_{V}$. Then this solution satisfies either (3.43) or (3.44). Assume first that this solution satisfies (3.43). Then a proof similar to that of Proposition 3.4 shows that since $V \leq V_{1}^{-}$, the following estimate holds:

$$
I \geq \mu_{+} \max \left(n_{1}+p_{1}, n_{-1}+p_{-1}\right)\left[V-\left(U_{b i}+2\left(N_{+}-N_{-}\right) \varepsilon^{-1}\right)\right],
$$

where $U_{b i}$, and $\mu_{+}$are respectively defined by (2.45), and (3.19). Assume now that $\left(\psi, n, p, J_{n}, I\right)$ is a solution of $(\mathrm{VD})_{V}$ such that (3.44) holds. Then a proof similar to that of Proposition 3.5 shows that the following estimate holds:

$$
\psi^{\prime}(x) \leq 2^{-1}\left(U_{b i}+6\left(N_{+}-N_{-}\right) / \varepsilon-V\right) \quad \forall x \in\left[\min \left(X_{m}^{-}, X_{e}^{-}\right), \max \left(X_{m}^{-}, X_{e}^{-}\right)\right],
$$

where $X_{e}^{-}$and $X_{m}^{-}$are defined as above. Two subcases can now occur as for the case $V \geq 0$ : either $s_{-}^{\prime}$ changes sign in $\left(\min \left(X_{m}^{-}, X_{e}^{-}\right), \max \left(X_{m}^{-}, X_{e}^{-}\right)\right)$or it keeps a constant sign in this interval. If $s_{-}^{\prime}$ changes sign in $\left(\min \left(X_{m}^{-}, X_{e}^{-}\right), \max \left(X_{m}^{-}, X_{e}^{-}\right)\right)$, then a proof similar to that of Proposition 3.6 shows that, since we have $V \leq V_{2}^{-}$, the following estimate holds:

$$
I \geq \mu_{+} \max \left(n_{-1}, p_{1}\right)\left[V-\left(U_{b i}+6\left(N_{+}-N_{-}\right) / \varepsilon\right)\right],
$$

where $\mu_{+}$is defined by (3.19). If $s_{-}^{\prime}$ keeps a constant sign in

$$
\left[\min \left(X_{m}^{-}, X_{e}^{-}\right), \max \left(X_{m}^{-}, X_{e}^{-}\right)\right],
$$

two subcases can occur: either (3.45) or (3.46) holds. If (3.45) holds, then a proof similar to that of Proposition 3.7 shows that since $V \leq V_{2}^{-}$holds, the following estimate is satisfied:

$$
I \geq 2^{-1} \mu_{+}\left[\max \left(2 n_{-1}-N_{-}, 2 p_{1}+N_{+}\right)\left(V-\left(U_{b i}\right)-\left(N_{+}-N_{-}\right)\right],\right.
$$

where $\mu_{+}$is defined by (3.19). If (3.46) holds, then a proof similar to that of Proposition 3.8 shows that since $V \leq V_{2}^{-}$holds, the following estimate is satisfied:

$$
I \geq 2^{-1} \mu_{+} \max \left(2 n_{-1}-N_{-}, 2 p_{1}+N_{+}\right)\left[V-\left(U_{b i}+6\left(N_{+}-N_{-}\right) / \varepsilon\right)\right],
$$

where $\mu_{+}$is defined by (3.19).

From all these cases we deduce that (3.40) holds with $V_{2}^{-}$given by (2.76), and $a_{1}^{-}>0$ and $a_{2}^{-}$given respectively by

$$
a_{1}^{-}=a_{1}^{+}
$$


where $a_{1}^{+}$is given by (3.38) and,

$$
a_{2}^{-}=-a_{1}^{-} U_{b i}-\left(N_{+}-N_{-}\right) \varepsilon^{-1} \max \left(2^{-1} \mu_{+} \varepsilon, 6 a_{1}^{-}\right) .
$$

This concludes the proof of the lemma.

Lemmas 2.9, 2.16 together with Lemmas 3.2 and 3.9 lead to the following corollary.

Corollary 3.10. Assume that the hypotheses of Theorem 2.7 hold. Then, every solution $\left(\psi, n, p, J_{n}, I\right)$ of $(\mathrm{VD})_{V}$ satisfies

$$
|I|=\mathcal{O}(|V|) \quad \text { as }|V| \rightarrow+\infty .
$$

The purpose of the next two lemmas is to prove a monotonicity property of the electron (resp. hole) current density and a local monotonicity property of the electric field at the ohmic contacts with respect to the total current $I$.

Lemma 3.11. (Monotonicity of the carrier current densities with respect to the total current). Assume that the hypotheses of Corollary 2.17 hold. Then the following property is satisfied:

$$
I<\tilde{I} \Rightarrow J_{n}<\tilde{J}_{n} \quad \text { and } \quad I-J_{n}<\tilde{I}-\tilde{J}_{n}, \quad \forall(I, \tilde{I}) \in \mathbb{R}^{2},
$$

where $J_{n}$ (resp. $\tilde{J}_{n}$ ) is the electron current density corresponding to the unique solution of $(\mathrm{CD})_{I}\left(\right.$ resp. $\left.(\mathrm{CD})_{\tilde{I}}\right)$.

The proof of Lemma 3.11 is based on arguments similar to those of the proof of Theorem 2.1. Nevertheless, due to technical reasons, we need to modify the proof of Theorem 2.1 in order to prove Lemma 3.11. From now on and during the proof of the next lemma, we use the notation of the proof of Theorem 2.1.

Proof. Let $I$ and $\tilde{I}$ be given such that $I<\tilde{I}$. From Theorem 2.1, we know that $(\mathrm{CD})_{I}$ and $(\mathrm{CD})_{\tilde{I}}$ have respectively a unique solution. We denote by $\left(\psi, n, p, J_{n}\right)$ (resp. $\left.\left(\tilde{\psi}, \tilde{n}, \tilde{p}, \tilde{J}_{n}\right)\right)$ the unique solution of $(\mathrm{CD})_{I}\left(\operatorname{resp} .(\mathrm{CD})_{\tilde{I}}\right)$. Then, $(\psi-\tilde{\psi})$ is in $\mathcal{C}^{2}(\bar{\Omega})$ and satisfies

$$
\varepsilon(\psi-\tilde{\psi})^{\prime \prime}=(n-\tilde{n})-(p-\tilde{p}) \quad \text { on } \quad \bar{\Omega} .
$$

Moreover, since $\mu_{n}$ and $\mu_{p}$ are continuous on each subinterval $\left[a_{i}, a_{i+1}\right]$, we deduce that $(n-\tilde{n}) \exp (-\psi)$ and $(p-\tilde{p}) \exp (\psi)$ are piecewise continuously differentiable on $\bar{\Omega}$. These functions satisfy respectively, on each subinterval $\left[a_{i}, a_{i+1}\right]$

$$
((n-\tilde{n}) \exp (-\psi))^{\prime}=\tilde{n} \exp (-\psi)(\psi-\tilde{\psi})^{\prime}+\left(J_{n}-\tilde{J}_{n}\right) \mu_{n}^{-1} \exp (-\psi),
$$

and,

$$
((p-\tilde{p}) \exp (\psi))^{\prime}=-\tilde{p} \exp (\psi)(\psi-\tilde{\psi})^{\prime}-\left(\left(I-J_{n}\right)-\left(\tilde{I}-\tilde{J}_{n}\right)\right) \mu_{p}^{-1} \exp (\psi)
$$

In order to prove (3.52), we proceed by contradiction. Assume that (3.52) does not hold. Then, since $I-\tilde{I}<0$ holds, we have either

$$
J_{n}-\tilde{J}_{n}<0 \quad \text { and } \quad 0 \leq\left(I-J_{n}\right)-\left(\tilde{I}-\tilde{J}_{n}\right),
$$

or

$$
0 \leq J_{n}-\tilde{J}_{n} \quad \text { and } \quad\left(I-J_{n}\right)-\left(\tilde{I}-\tilde{J}_{n}\right)<0
$$


Let us first assume that (3.56) holds. Of course, since (3.54), $J_{n}-\tilde{J}_{n}<0$ and $(n-\tilde{n})( \pm 1)=0$ hold, $(\psi-\tilde{\psi})^{\prime}$ cannot be identically zero on $\Omega$. We now proceed as for the proof of Theorem 2.1. We define $X$ as in the proof of Theorem 2.1. Then, a proof similar to that of Proposition 2.2 shows that, since (3.56) holds, $X \cap \Omega \neq \emptyset$. Therefore, we can define $\alpha$ and $\beta$ as in the proof of Theorem 2.1. A proof similar to that of Proposition 2.4 shows that, since (3.56) holds, the inequalities (2.9) and (2.10) still hold, and that both inequalities of (2.9) (resp. of (2.10)) are strict inequalities, if $\alpha \neq-1$ (resp. $\beta \neq 1$ ) holds.

We claim, as in the proof of Theorem 2.1, that $\alpha=\beta$ holds. To the contrary, assume that $\alpha \neq \beta$ holds. We define $Y$ as in the proof of Theorem 2.1. Then, since $\alpha<\beta$ and (2.10) hold, $Y$ is not empty, so that we can define $\gamma=\inf (Y)$. Of course (2.12) and $\gamma \in X \cap[\alpha, 1]$ still hold, as in the proof of Theorem 2.1. Moreover, since $J_{n}-\tilde{J}_{n}<0$ holds, we still have

$$
\lim _{h \rightarrow 0^{+}}((n-\tilde{n}) \exp (-\psi))^{\prime}(\alpha+h)<0 .
$$

If $0<\left(I-J_{n}\right)-\left(\tilde{I}-\tilde{J}_{n}\right)$ holds, then we have

$$
\lim _{h \rightarrow 0^{+}}((p-\tilde{p}) \exp (\psi))^{\prime}(\alpha+h)<0,
$$

so that, as in the proof of Theorem 2.1, we have $\alpha<\gamma$. Moreover, if (3.56) and $0<\left(I-J_{n}\right)-\left(\tilde{I}-\tilde{J}_{n}\right)$ hold, it is easy to check that the results of Propositions 2.5 and 2.6 still hold. Therefore, we conclude, as in the proof of Theorem 2.1, that the case where $(3.56)$ and $0<\left(I-J_{n}\right)-\left(\tilde{I}-\tilde{J}_{n}\right)$ hold, leads to a contradiction. Hence, we can assume now that $(3.56)$ and

$$
\left(I-J_{n}\right)-\left(\tilde{I}-\tilde{J}_{n}\right)=0,
$$

hold. Of course, in this case, (3.59) no longer holds. We claim that $\alpha<\gamma$ still holds. Two subcases can occur: either $\alpha \neq-1$ or $\alpha=-1$. If $\alpha \neq-1$ holds, then (2.9) holds with strict inequalities. Since (2.12) also holds, we deduce that if $\alpha \neq-1$ holds, then we have $\alpha<\gamma$. Assume now that $\alpha=-1$. Then we have $(\psi-\tilde{\psi})^{\prime}(-1)=0$. This, together with $(3.55)$ and $(3.60)$ imply that $(p-\tilde{p})^{\prime}(-1)=0$. On the other hand, since $n-\tilde{n}$ and $p-\tilde{p}$ are piecewise continuously differentiable on $\bar{\Omega}$, we deduce that $(\psi-\tilde{\psi})^{\prime \prime}$ is piecewise continuously differentiable on $\bar{\Omega}$. Therefore, differentiating (3.53), setting $x=-1$ in the resulting equation, and using the equation $(p-\tilde{p})^{\prime}(-1)=0$, we deduce that

$$
\varepsilon(\psi-\tilde{\psi})^{(3)}(-1)=(n-\tilde{n})^{\prime}(-1) .
$$

This, together with $(3.58)$ and $(n-\tilde{n})(-1)=0$, imply that

$$
(\psi-\tilde{\psi})^{(3)}(-1)<0 .
$$

Since $(\psi-\tilde{\psi})^{\prime \prime}$ is continuously differentiable in a right neighbourhood of $x=-1$, and since $(\psi-\tilde{\psi})^{\prime}(-1)=(\psi-\tilde{\psi})^{\prime \prime}(-1)=0$, we deduce that

$$
\exists \eta>0 \text { such that }(\psi-\tilde{\psi})^{\prime}<0 \text { on }(-1,-1+\eta] .
$$

This, together with $\alpha=-1$, imply in particular that

$$
X-[-1, \alpha] \subset(-1+\eta, 1],
$$

so that we have $Y \subset(-1+\eta, 1]$. From this, we deduce that $\alpha<\gamma$. Therefore, we proved that if $\alpha=-1$, then $\alpha<\gamma$ still holds. Thus, for both subcases $\alpha \neq-1$ or $\alpha=-1$, we have $\alpha<\gamma$. Then using (3.56), we prove as in Proposition 2.5, that 
if (2.15) holds, then $X \cap(z, \gamma) \neq \emptyset$. From this, we deduce as in Proposition 2.6, that there exists a sequence $\left(z_{i}\right)_{i \in \mathbb{N}}$ of points of $(\alpha, \gamma) \cap X$ satisfying (2.16)-(2.17). Hence, we have

$$
(n-\tilde{n})(\gamma) \leq 0, \quad(p-\tilde{p})(\gamma) \leq 0 .
$$

Since (2.12) holds, we deduce as in the proof of Theorem 2.1 that

$$
(n-\tilde{n})(\gamma)=0 \quad \text { or } \quad(p-\tilde{p})(\gamma)=0 .
$$

If $(n-\tilde{n})(\gamma)=0$, then we proceed exactly as in the proof of Theorem 2.1 and we obtain a contradiction since (3.58) holds. Hence, we can assume that $(n-\tilde{n})(\gamma) \neq 0$ holds, so that we have now $(p-\tilde{p})(\gamma)=0$. Using now $(3.60)$ and $(p-\tilde{p})(\gamma)=0$ in $(3.55)$, we deduce that $(p-\tilde{p})^{\prime}(\gamma)=0$. Now, since we have $(n-\tilde{n})(\gamma) \neq 0$ and $(n-\tilde{n})(\gamma) \leq 0$, we deduce that $(n-\tilde{n})(\gamma)<0$ holds. This, together with $(p-\tilde{p})(\gamma)=0$ and $(3.53)$, imply that

$$
(\psi-\tilde{\psi})^{\prime \prime}(\gamma)<0
$$

Since $\gamma \in X$, we deduce that

$$
\exists \eta>0 \quad \text { such that }(\psi-\tilde{\psi})^{\prime}>0 \text { on }[\gamma-\eta, \gamma) .
$$

But, this is impossible, since the sequence $\left(z_{i}\right)_{i \in \mathbb{N}}$ satisfies $(2.17)$ and

$$
z_{i} \in X \cap(\alpha, \gamma) \quad \forall i \in \mathbb{N},
$$

Therefore, we proved that if (3.56) and (3.60) hold, then we have a contradiction. This proves that $\alpha=\beta$, so that we have $X=\{\alpha\}$. We then conclude as in the proof of Theorem 2.1, that we have a contradiction. This proves that the case where (3.56) and (3.60) hold, is impossible. Therefore, we proved that (3.56) cannot hold.

The case where (3.57) holds, can be treated in a similar way (in this case, we permute the two solutions $\left(\psi, n, p, J_{n}\right)$ and $\left(\tilde{\psi}, \tilde{n}, \tilde{p}, \tilde{J}_{n}\right)$ and we consider the two subcases $J_{n}-\tilde{J}_{n} \neq 0$ or $\left.J_{n}-\tilde{J}_{n}=0\right)$. This proves that (3.52) holds and concludes the proof of the lemma.

The following lemma shows that the electric field at the ohmic contacts $x= \pm 1$, is a strictly increasing function of the total current $I$.

Lemma 3.12. (Local monotonicity of the electric field with respect to the total current). Assume that the hypotheses of Corollary 2.17 hold. Then the following property is satisfied:

$$
I<\tilde{I} \Rightarrow 0<(\psi-\tilde{\psi})^{\prime}(1) \quad \text { and } \quad 0<(\psi-\tilde{\psi})^{\prime}(-1) \quad \forall(I, \tilde{I}) \in \mathbb{R}^{2},
$$

where $\psi($ resp. $\tilde{\psi})$ is the electrostatic potential corresponding to the unique solution of $(\mathrm{CD})_{I}\left(\right.$ resp. $\left.(\mathrm{CD})_{\tilde{I}}\right)$.

Proof. Let $I$ and $\tilde{I}$ be given such that $I<\tilde{I}$. We denote by $\left(\psi, n, p, J_{n}\right)$ (resp. $\left.\left(\tilde{\psi}, \tilde{n}, \tilde{p}, \tilde{J}_{n}\right)\right)$ the unique solution of $(\mathrm{CD})_{I}\left(\right.$ resp. $\left.(\mathrm{CD})_{\tilde{I}}\right)$ and we keep the notations of the proof of Lemma 3.11. In order to prove (3.62) we proceed by contradiction. Assume that (3.62) does not hold. This is equivalent to assume that we have

$$
(\psi-\tilde{\psi})^{\prime}(1) \leq 0 \quad \text { or } \quad(\psi-\tilde{\psi})^{\prime}(-1) \leq 0 .
$$

On the other hand, since we have $I-\tilde{I}<0$, we deduce from Lemma 3.11 that (3.52) holds. Let us first assume that the first inequality of (3.63) holds. Two subcases 
can occur: either $(\psi-\tilde{\psi})^{\prime}(1)=0$, or $(\psi-\tilde{\psi})^{\prime}(1)<0$ holds. We claim that for both subcases, we have

$$
(\psi-\tilde{\psi})^{\prime} \leq 0 \quad \text { in a left neighbourhood of } 1 .
$$

Indeed assume first that we have $(\psi-\tilde{\psi})^{\prime}(1)=0$. From (1.1), we deduce that we have

$$
\varepsilon(\psi-\tilde{\psi})^{\prime \prime}=(n-\tilde{n})-(p-\tilde{p}) \quad \text { on } \quad \Omega .
$$

Since $n-\tilde{n}$ and $p-\tilde{p}$ are in $\mathcal{C}^{1}(\bar{\Omega})$, we can differentiate the above equation. We use then (1.2) and (1.3) in the resulting equation, set $x=1$ in the final equation and use the equation $(\psi-\tilde{\psi})^{\prime}(1)=0$. We finally obtain

$$
\varepsilon(\psi-\tilde{\psi})^{(3)}(1)=I-\tilde{I}<0
$$

Setting now $x=1$ in (3.65) and using the above inequality, we obtain (3.64). If $(\psi-\tilde{\psi})^{\prime}(1)<0$ holds, then (3.64) clearly holds. Hence we proved that if the first inequality of (3.63) holds, then (3.64) holds. Using now the first inequality of (3.52) in (3.54), together with the boundary conditions $(n-\tilde{n})( \pm 1)=0$ and (3.63), we deduce that $(\psi-\tilde{\psi})^{\prime}$ cannot keep a constant sign in $\Omega$. Hence, there exists $z_{K}$ in $\Omega$, such that $(\psi-\tilde{\psi})^{\prime}$ changes sign at $x=z_{K}$, and such that $(\psi-\tilde{\psi})^{\prime}$ keeps a constant sign in $\left[z_{K}, 1\right]$ and is not identically zero in this interval. From (3.64), we deduce that

$$
(\psi-\tilde{\psi})^{\prime} \leq 0 \quad \text { in } \quad\left[z_{K}, 1\right] .
$$

Using (3.66) and the first inequality of (3.52) (resp. the second inequality of (3.52)) in (3.54) (resp. (3.55)) we obtain

$$
\begin{gathered}
((n-\tilde{n}) \exp (-\psi))^{\prime}<0 \quad \text { in } \quad\left[z_{K}, 1\right], \\
\text { (resp. } \left.-((p-\tilde{p}) \exp (\psi))^{\prime}<0 \quad \text { in }\left[z_{K}, 1\right]\right) .
\end{gathered}
$$

From the boundary conditions $(n-\tilde{n})(1)=(p-\tilde{p})(1)=0$, we deduce that

$$
0<(n-\tilde{n})(x) \quad \text { and } \quad 0<-(p-\tilde{p})(x) \quad \forall x \in\left[z_{K}, 1\right] .
$$

This together with (3.65) imply

$$
(\psi-\tilde{\psi})^{\prime}\left(z_{K}\right)<(\psi-\tilde{\psi})^{\prime}(1) .
$$

This is impossible, since we have $(\psi-\tilde{\psi})^{\prime}\left(z_{K}\right)=0$, whereas $(\psi-\tilde{\psi})^{\prime}(1) \leq 0$. In a similar way, we prove that the second inequality of (3.63) cannot hold (in this case, we prove that there exists $z_{1}$ in $\Omega$, such that $(\psi-\tilde{\psi})^{\prime}$ changes sign at $x=z_{1}$, and such that $(\psi-\tilde{\psi})^{\prime}$ keeps a constant sign in $\left[-1, z_{1}\right]$ and we consider the interval $\left[-1, z_{1}\right]$, instead of the interval $\left.\left[z_{K}, 1\right]\right)$. This proves that (3.63) cannot hold. Hence (3.62) holds, which concludes the proof of the lemma.

\section{Comparison With the QUASI-NEUTRAL APPROXIMATION}

For most realistic devices, the doping profile $N$ is a piecewise smooth function, and the parameter $\varepsilon$ which appears in (1.1) is very small compared to 1 . Therefore, both systems $(\mathrm{VD})_{V}$ and $(\mathrm{CD})_{I}$ can be studied in the context of singular perturbation theory (see e.g. [11], [3]). The quasi-neutral approximation of the voltage (resp. current) driven model is derived from $(\mathrm{VD})_{V}\left(\right.$ resp. $\left.(\mathrm{CD})_{I}\right)$ by setting $\varepsilon=0$ in (1.1) and by adding interface conditions at the points where $N$ is discontinuous. The resulting system of equation obtained under this approximation is called 
the reduced voltage (resp. current) driven model and is denoted from now on by $(\mathrm{RVD})_{V}\left(\right.$ resp. $\left.(\mathrm{RCD})_{I}\right)$.

If $\left(\psi, n, p, J_{n}, I\right)\left(\operatorname{resp} .\left(\bar{\psi}, \bar{n}, \bar{p}, \overline{J_{n}}, \bar{I}\right)\right)$ is a solution of $(\mathrm{VD})_{V}\left(\operatorname{resp} .(\mathrm{RVD})_{V}\right)$, we call $I$ (resp. $\bar{I}$ ) the full (resp. reduced) current corresponding to the solution of $(\mathrm{VD})_{V}\left(\right.$ resp. $\left.(\mathrm{RVD})_{V}\right)$ under consideration.

The purpose of this section is to show through the particular class of symmetric devices, that the quasi-neutral approximation is not accurate for large $|V|$, this even if no saturation phenomenon occurs for the reduced currents. This section is organized as follows. We first give the Uniqueness theorem 4.1 for $(\mathrm{RCD})_{I}$. Then, we establish a symmetry result for symmetric devices in Lemma 4.2 and we give a necessary and sufficient condition on $N$ and $I$ for the nonexistence of solutions of $(\mathrm{RCD})_{I}$ in Theorem 4.4. Finally, we compare the full and the reduced voltage driven models in Corollary 4.5.

For the sake of simplicity, we restrict our analysis to the case of piecewise analytic $N, \mu_{n}$ and $\mu_{p}$. Hence we assume from now on that $N, \mu_{n}$ and $\mu_{p}$ satisfy

$$
\begin{aligned}
& \exists\left(a_{i}\right)_{1 \leq i \leq M} \in(\Omega)^{M}, \text { such that } a_{i}<a_{i+1}, a_{0}=-1, a_{M+1}=1 \text { and } \\
& N, \mu_{n}, \mu_{p} \text { are analytic on }\left[a_{i}, a_{i+1}\right] \quad \forall i \in\{0, \ldots, M\} .
\end{aligned}
$$

Moreover we set for all the sequel of this paper

$$
\Gamma=\bigcup_{i=1}^{i=M}\left\{a_{i}\right\}, \quad \Omega^{\prime}=\Omega-\Gamma .
$$

If the semiconductor under consideration is symmetric, the following relation

$$
\psi(-1)+\psi(1)=0
$$

is more appropriate than the boundary condition (1.5). Since the potential is defined up to an additive constant, there is no loss of generality, when we replace (1.5) by $(4.2)$ in $(\mathrm{VD})_{V}$ and $(\mathrm{CD})_{I}$. We denote by $(\mathrm{VD})_{V}^{\prime}$ and $(\mathrm{CD})_{I}^{\prime}$ the corresponding modified voltage and current driven systems. Moreover, one can easily check that the results of the previous sections are still valid for $(\mathrm{VD})_{V}^{\prime}$ and $(\mathrm{CD})_{I}^{\prime}$, provided that $V$ is replaced by $2 V$ in the estimates of Lemmas $2.9,2.16,3.2,3.9$ and that (1.12) is replaced by

$$
I=\delta^{2}(\exp (V)-\exp (-V))\left(\left(\int_{\Omega} \exp (-\psi) \mu_{n}^{-1} d x\right)^{-1}+\left(\int_{\Omega} \exp (\psi) \mu_{p}^{-1} d x\right)^{-1}\right) .
$$

For a given current $I$, the reduced current driven model $(\mathrm{RCD})_{I}$ is:

$$
\begin{gathered}
n-p-N=0 \text { in } \Omega^{\prime}, \\
\mu_{n}\left(n^{\prime}-n \psi^{\prime}\right)=J_{n} \text { in } \Omega^{\prime}, \\
\mu_{p}\left(p^{\prime}+p \psi^{\prime}\right)=J_{n}-I \text { in } \Omega^{\prime}, \\
J_{n}^{\prime}=0 \text { in } \Omega^{\prime},
\end{gathered}
$$

subject to the boundary conditions $(4.2),(1.6),(1.7)$ and to the interface conditions

$$
\left[J_{n}\right]_{a_{i}}=0,[n \exp (-\psi)]_{a_{i}}=0,[p \exp (\psi)]_{a_{i}}=0 \quad \forall i \in\{1, \ldots, M\},
$$


where $[f]_{a_{i}}=f\left(a_{i}^{+}\right)-f\left(a_{i}^{-}\right)$denotes the jump of the function $\mathrm{f}$ at the point $a_{i}$. The unknowns of (RCD) $I$ are $\psi, n, p$ and $J_{n}$.

For a given $V$, the reduced voltage driven model $(\mathrm{RVD})_{V}$ consists of the system (4.4)-(4.7) and

$$
I^{\prime}=0 \text { in } \Omega^{\prime},
$$

subject to the boundary conditions $(4.2),(1.6)-(1.10)$ and to the interface conditions (4.8) and

$$
[I]_{a_{i}}=0 \quad \forall i \in\{1, \ldots, M\} .
$$

In this case the unknowns are $\left(\psi, n, p, J_{n}, I\right)$. We recall that under the assumption (4.1), (RVD) $V$ has at least one weak solution for all $V$ in $\mathbb{R}$ (see [11] for the proof). Moreover any weak solution $\left(\psi, n, p, J_{n}, I\right)$ of $(\mathrm{RVD})_{V}$ satisfies

$$
0<n\left(x^{ \pm}\right), 0<p\left(x^{ \pm}\right) \quad \forall x \in \bar{\Omega} .
$$

In addition, we remark that if $\left(\psi, n, p, J_{n}, I\right)$ (resp. $\left.\left(\psi, n, p, J_{n}\right)\right)$ is a weak solution of $(\mathrm{RVD})_{V}\left(\right.$ resp. $\left.(\mathrm{RCD})_{I}\right)$, then $w=n+p$ satisfies the following equation in $\Omega^{\prime}$ :

$$
w^{\prime}=w^{-1} N\left(N^{\prime}-\left(J_{n} \mu_{n}^{-1}+\left(I-J_{n}\right) \mu_{p}\right)\right)+J_{n} \mu_{n}^{-1}-\left(I-J_{n}\right) \mu_{p} .
$$

Hence, under the assumption (4.1), it follows from classical results on ordinary differential equations (see [7]) that $\psi, n$ and $p$ are analytic on each subinterval $\left[a_{i}, a_{i+1}\right]$.

We can now state the following uniqueness result:

Theorem 4.1. Assume that $N, \mu_{n}, \mu_{p}$ satisfy (4.1) and that $\mu_{n}$ and $\mu_{p}$ satisfy (1.11). Then for every $I$ in $\mathbb{R},(\mathrm{RCD})_{I}$ has at most one weak solution.

Proof. Assume that $I$ is such that $(\mathrm{RCD})_{I}$ has two weak solutions $\left(\psi, n, p, J_{n}\right)$ and $\left(\tilde{\psi}, \tilde{n}, \tilde{p}, \tilde{J}_{n}\right)$. We set

$$
u_{i}=(\psi-\tilde{\psi})_{\mid\left[a_{i}, a_{i+1}\right]} \quad \forall i \in\{0, \ldots, M\} .
$$

We claim that the following property holds:

$$
\begin{aligned}
& \forall i \in\{1, \ldots, M\} \quad \exists r_{i}>0 \text { such that }(n-\tilde{n})\left(a_{i}^{+}\right)=r_{i}(n-\tilde{n})\left(a_{i}^{-}\right), \\
& \text {and }(p-\tilde{p})\left(a_{i}^{+}\right)=r_{i}(p-\tilde{p})\left(a_{i}^{-}\right) .
\end{aligned}
$$

In order to prove (4.9), we proceed as follows: Since (4.4) holds, we have

$$
(n-p)\left(x^{ \pm}\right)=(\tilde{n}-\tilde{p})\left(x^{ \pm}\right)=N\left(x^{ \pm}\right) \quad \forall x \in \bar{\Omega} .
$$

This implies that

$$
(n+p)^{2}\left(a_{i}^{ \pm}\right)=N^{2}\left(a_{i}^{ \pm}\right)+4(n p)\left(a_{i}^{ \pm}\right) \quad \forall i \in\{1, \ldots, M\} .
$$

In a similar way, we deduce that

$$
(\tilde{n}+\tilde{p})^{2}\left(a_{i}^{ \pm}\right)=N^{2}\left(a_{i}^{ \pm}\right)+4(\tilde{n} \tilde{p})\left(a_{i}^{ \pm}\right) \quad \forall i \in\{1, \ldots, M\} .
$$

On the other hand, using now the two last equations of (4.8), we obtain

$$
[n p]_{a_{i}}=[\tilde{n} \tilde{p}]_{a_{i}}=0 \quad \forall i \in\{1, \ldots, M\} .
$$

This, together with (4.11) and (4.12) give

$$
(n+p)^{2}\left(a_{i}^{+}\right)-(\tilde{n}+\tilde{p})^{2}\left(a_{i}^{+}\right)=(n+p)^{2}\left(a_{i}^{-}\right)-(\tilde{n}+\tilde{p})^{2}\left(a_{i}^{-}\right) \quad \forall i \in\{1, \ldots, M\} .
$$

Since $n+p$ and $\tilde{n}+\tilde{p}$ are strictly positive functions on $\bar{\Omega}$, we deduce that

$$
(n+p)\left(a_{i}^{+}\right)-(\tilde{n}+\tilde{p})\left(a_{i}^{+}\right)=r_{i}\left((n+p)\left(a_{i}^{-}\right)-(\tilde{n}+\tilde{p})\left(a_{i}^{-}\right)\right) \quad \forall i \in\{1, \ldots, M\},
$$


where $r_{i}>0$ is given by

$$
\frac{(n+p)\left(a_{i}^{-}\right)+(\tilde{n}+\tilde{p})\left(a_{i}^{-}\right)}{(n+p)\left(a_{i}^{+}\right)+(\tilde{n}+\tilde{p})\left(a_{i}^{+}\right)} .
$$

From this and (4.10), we get (4.9). Hence, we proved our claim.

Using now (4.7) and the first equation of (4.8), we deduce that $J_{n}-\tilde{J}_{n}$ is constant on $\bar{\Omega}$. Hence, we can assume without loss of generality that

$$
J_{n}-\tilde{J}_{n} \leq 0 \text {. }
$$

Moreover, since $u_{i}$ is analytic on each subinterval $\left[a_{i}, a_{i+1}\right]$, we can define the set

$$
Z=\left\{z \in \Omega^{\prime},(\psi-\tilde{\psi})^{\prime} \text { changes sign at } x=z\right\} .
$$

Of course this set is finite. Assume first that $Z$ is not empty. We denote by $\left(z_{i}\right)_{1 \leq i \leq K}$ its elements, ordered in a strictly increasing sequence. Then, we claim that the following inequalities hold:

$$
(n-\tilde{n})(z)<0,(p-\tilde{p})(z)<0 \quad \forall z \in Z,
$$

and,

$$
(n-\tilde{n})(z) \leq 0,(p-\tilde{p})(z) \leq 0 \quad \forall z \in \Gamma .
$$

In order to prove (4.15)-(4.16), we first prove by induction on $i \in\{1, \ldots, K\}$, the following inequalities:

$$
(n-\tilde{n})(z)<0,(p-\tilde{p})(z)<0 \quad \forall z \in\left\{z_{1}, \ldots, z_{i}\right\},
$$

and,

$$
(n-\tilde{n})(z) \leq 0,(p-\tilde{p})(z) \leq 0 \quad \forall z \in A_{i}=\left\{a_{j}, a_{j}<z_{i}\right\} .
$$

Let us first prove that (4.17)-(4.18) holds for $i=1$. Two cases can occur: either $A_{1}=\emptyset$ or $A_{1} \neq \emptyset$.

Case (1). Assume that $A_{1}=\emptyset$.

This implies that $z_{1} \in\left(-1, a_{1}\right)$. By definition of $z_{1}, u_{0}^{\prime}$ keeps a constant sign in $\left[-1, z_{1}\right]$. On the other hand, we deduce from (4.5) and (4.6) respectively, that

$$
((n-\tilde{n}) \exp (-\psi))^{\prime}=\tilde{n} \exp (-\psi)(\psi-\tilde{\psi})^{\prime}+\left(J_{n}-\tilde{J}_{n}\right) \mu_{n}^{-1} \exp (-\psi), \text { in } \quad \Omega^{\prime},
$$

and,

$$
((p-\tilde{p}) \exp (\psi))^{\prime}=-\tilde{p} \exp (\psi)(\psi-\tilde{\psi})^{\prime}+\left(J_{n}-\tilde{J}_{n}\right) \mu_{p}^{-1} \exp (\psi) \text { in } \Omega^{\prime} .
$$

Hence, if $u_{0}^{\prime} \leq 0$ (resp. $\left.\geq 0\right)$ in $\left[-1, z_{1}\right]$, we use (4.19) (resp. (4.20)), (4.14) and the boundary conditions $(n-\tilde{n})(-1)=0$ (resp. $(p-\tilde{p})(-1)=0)$ and $u_{0}^{\prime} \neq 0$ in $\left[-1, z_{1}\right]$ and deduce that

$$
(n-\tilde{n})\left(z_{1}\right)<0 \quad\left(\operatorname{resp} . \quad(p-\tilde{p})\left(z_{1}\right)<0\right) .
$$

Using (4.10) and the above inequality, we deduce that for both cases $u_{0}^{\prime} \leq 0$ or $u_{0}^{\prime} \geq 0$ in $\left[-1, z_{1}\right]$, we have

$$
(n-\tilde{n})\left(z_{1}\right)=(p-\tilde{p})\left(z_{1}\right)<0 .
$$

This proves that if $A_{1}=\emptyset$, then (4.17)-(4.18) holds for $i=1$ and concludes the case (1). 
Case (2). Assume now that $A_{1} \neq \emptyset$.

We denote by $a_{k}$ the largest element of $A_{1}$. Hence, by definition of $z_{1}, u_{i}^{\prime}$ keeps a constant sign on $\left[a_{i}, a_{i+1}\right]$ for all $i \in\{0, \ldots, k-1\}$. In particular, since $u_{0}^{\prime}$ keeps a constant sign on $\left[-1, a_{1}\right]$, we get by using the appropriate equation (4.19) or (4.20)

$$
(n-\tilde{n})\left(a_{1}^{-}\right) \leq 0,(p-\tilde{p})\left(a_{1}^{-}\right) \leq 0 .
$$

Setting now $i=1$ in (4.9) and using the above inequalities, we obtain

$$
(n-\tilde{n})\left(a_{1}^{+}\right) \leq 0,(p-\tilde{p})\left(a_{1}^{+}\right) \leq 0 .
$$

In the same way, we prove easily by induction on $j$ that the following inequalities hold:

$$
(n-\tilde{n})\left(a_{j}^{ \pm}\right) \leq 0,(p-\tilde{p})\left(a_{j}^{ \pm}\right) \leq 0 \quad \forall j \in\{1, \ldots, k\} .
$$

This proves that (4.18) holds for $i=1$. Moreover, we deduce, in particular from (4.21) that

$$
(n-\tilde{n})\left(a_{k}^{+}\right) \leq 0,(p-\tilde{p})\left(a_{k}^{+}\right) \leq 0 .
$$

On the other hand, $u_{k}^{\prime}$ keeps a constant sign on $\left[a_{k}, z_{1}\right]$ and is not identically zero on this interval. This, together with (4.22) and the appropriate equation (4.19) or (4.20) give

$$
(n-\tilde{n})\left(z_{1}\right)<0,(p-\tilde{p})\left(z_{1}\right)<0 .
$$

Hence we proved that if $A_{1} \neq \emptyset$, then (4.17)-(4.18) hold for $i=1$. This concludes the case (2). Hence we proved that for both cases, (4.17)-(4.18) hold for $i=1$. Let us now assume that (4.17)-(4.18) hold for all $i \in\{1, \ldots, k\}$ and let us prove that (4.17)-(4.18) also hold for $i=k+1$. Since the proof is similar to that of the case $i=1$, we only sketch it. As for the case $i=1$, two cases can occur: either $A_{k+1}=A_{k}$ or $A_{k+1} \neq A_{k}$. The first case implies that $\Gamma \cap\left(z_{k}, z_{k+1}\right)=\emptyset$ and is similar to the case $A_{1}=\emptyset$. The second case implies that $\Gamma \cap\left(z_{k}, z_{k+1}\right) \neq \emptyset$ and is similar to the case $A_{1} \neq \emptyset$. This proves that (4.17)-(4.18) hold for $i=k+1$. Hence, we proved by induction on $i$, that (4.17)-(4.18) hold for all $i \in\{1, \ldots, K\}$.

We can now prove (4.15)-(4.16). Since (4.17)-(4.18) hold for $i=K$, we have

$$
(n-\tilde{n})\left(z_{K}\right)<0,(p-\tilde{p})\left(z_{K}\right)<0 .
$$

We denote by $a_{k}$ the largest element of $A_{K}$. Then, by definition of $z_{K}, u_{k}^{\prime}$ keeps a constant sign on $\left[z_{K}, a_{k+1}\right]$, whereas $u_{j}^{\prime}$ keeps a constant sign on $\left[a_{j}, a_{j+1}\right]$ for all $j \in\{k+1, \ldots, M\}$. From this, (4.23) and the appropriate equation (4.19) or (4.20), we deduce as before that

$$
(n-\tilde{n})\left(a_{j}^{ \pm}\right) \leq 0,(p-\tilde{p})\left(a_{j}^{ \pm}\right) \leq 0 \quad \forall j \in\{k+1, \ldots, M\} .
$$

This, together with (4.17)-(4.18) for $i=K$, give (4.15)-(4.16). Hence, we proved that if $Z$ is not empty, then (4.15)-(4.16) hold. But, we claim now that the following inequalities also hold:

$$
(n-\tilde{n})\left(z_{K}\right)>0,(p-\tilde{p})\left(z_{K}\right)>0 .
$$

In order to prove (4.24), we set $B_{K}=\left\{a_{j}, z_{K}<a_{j}\right\}$. Two cases can occur: either $B_{K}=\emptyset$ or $B_{K} \neq \emptyset$.

Assume first that $B_{K}=\emptyset$. This implies that $a_{M}<z_{K}$, hence $u_{M}^{\prime}$ keeps a constant sign on $\left[z_{K}, 1\right]$ and is not identically zero on this interval. Therefore, using the appropriate equation (4.19) or (4.20) and the appropriate boundary condition $(n-\tilde{n})(1)=0$ or $(p-\tilde{p})(1)=0$, we deduce that (4.24) holds. 
Assume now that $B_{K} \neq \emptyset$. We denote then by $a_{k}$, the smallest element of $B_{K}$. Hence, by definition of $z_{K}, u_{i}^{\prime}$ keeps a constant sign on $\left[a_{i}, a_{i+1}\right]$ for all $i \in$ $\{k, \ldots, M\}$. In particular, since $u_{M}^{\prime}$ keeps a constant sign on $\left[a_{M}, 1\right]$, we deduce, by using the appropriate equation (4.19) or (4.20) that

$$
(n-\tilde{n})\left(a_{M}^{+}\right) \geq 0,(p-\tilde{p})\left(a_{M}^{+}\right) \geq 0 .
$$

Setting $i=M$ in (4.9) and using the above inequalities, we get

$$
(n-\tilde{n})\left(a_{M}^{-}\right) \geq 0,(p-\tilde{p})\left(a_{M}^{-}\right) \geq 0 .
$$

In the same way, we prove easily by a backward induction on $i$ that

$$
(n-\tilde{n})\left(a_{i}^{ \pm}\right) \geq 0,(p-\tilde{p})\left(a_{i}^{ \pm}\right) \geq 0 \quad \forall i \in\{k, \ldots, M\} .
$$

Hence, we have in particular for $i=k$

$$
(n-\tilde{n})\left(a_{k}^{-}\right) \geq 0,(p-\tilde{p})\left(a_{k}^{-}\right) \geq 0 .
$$

Now, using the fact that $u_{k-1}^{\prime}$ keeps a constant sign on $\left[z_{K}, a_{k}\right]$ and is not identically zero on this interval, we get (4.24) as before.

Therefore we proved that for both cases $B_{K}=\emptyset$ or $B_{K} \neq \emptyset$, (4.24) holds. But, this is impossible, since (4.24) contradicts (4.15) for $z=z_{K}$. Hence we proved that the assumption $Z \neq \emptyset$ leads to a contradiction. Therefore $Z$ is necessarily empty, which implies that $u_{i}^{\prime}$ keeps a constant sign on $\left[a_{i}, a_{i+1}\right]$ for all $i \in\{0, \ldots, M\}$. Starting from $x=-1$ and using the appropriate equation (4.19) or (4.20), together with (4.9), we deduce that

$$
(n-\tilde{n})\left(z^{ \pm}\right) \leq 0,(p-\tilde{p})\left(z^{ \pm}\right) \leq 0 \quad \forall z \in \bar{\Omega} .
$$

But starting now from $x=1$, we deduce in the same way that

$$
(n-\tilde{n})\left(z^{ \pm}\right) \geq 0,(p-\tilde{p})\left(z^{ \pm}\right) \geq 0 \quad \forall z \in \bar{\Omega} .
$$

Combining these inequalities, we get

$$
(n-\tilde{n})\left(z^{ \pm}\right)=0,(p-\tilde{p})\left(z^{ \pm}\right)=0 \quad \forall z \in \bar{\Omega} .
$$

Using these last equations in (4.19) and (4.20), we obtain

$$
u_{i}^{\prime}=0 \text { in }\left[a_{i}, a_{i+1}\right] \quad \forall i \in\{0, \ldots, M\}, \quad J_{n}-\tilde{J}_{n}=0 .
$$

Setting now $z=a_{i}$ in the first equation of (4.25) and using the resulting equation in the first relation of (4.8), we get

$$
[\psi-\tilde{\psi}]_{a_{i}}=0 \quad \forall i \in\{1, \ldots, M\} .
$$

This, together with the first equality of (4.26) imply that there exists $c \in \mathbb{R}$ such that $(\psi-\tilde{\psi})(x)=c$ for all $x \in \bar{\Omega}$. Using now (4.2), we finally obtain $c=0$. This proves that the two solutions $\left(\psi, n, p, J_{n}\right)$ and $\left(\tilde{\psi}, \tilde{n}, \tilde{p}, \tilde{J}_{n}\right)$ coincide on $\bar{\Omega}$, which concludes the proof of the theorem.

The purpose of the next results is twofold. First, we show that the existence result obtained in section 2 for the current driven model is not preserved in general when one considers the reduced current driven model. In particular, we give an explicit necessary and sufficient condition on the doping profile $N$ for the occurrence of saturation currents, in the case of symmetric devices. Then, we show that, even when no saturation phenomenon occurs, the quasi-neutral approximation is not accurate, in general, for large reverse and forward biases. In particular, we prove that for symmetric devices, the error between the full and the reduced currents goes to infinity as the $|V|$ goes to infinity. 
We now restrict our analysis of the quasi-neutral approximation to the case of symmetric devices. In this case, we show that the reduced current and voltage driven models have symmetry properties, which allow us to find an explicit relation of $V$ in terms of the reduced current. Hence, assume now that the device under consideration is symmetric. That is, the doping profile $N$ satisfies (4.1) with

$(4.27)$

$\exists K \in \mathbb{N}$ such that $M=2 K+1, \quad \exists\left(b_{i}\right)_{0 \leq i \leq K+1}$ such that

$a_{i}=-b_{K+1-i} \quad \forall i \in\{0, \ldots, K+1\}, a_{i}=b_{i-(K+1)} \quad \forall i \in\{K+2, \ldots, 2 K+2\}$,

$[N]_{b_{i}} \neq 0, \quad N(x)=-N(x) \forall x \in\left[b_{i}, b_{i+1}\right], \quad \forall i \in\{0, \ldots, K\}$.

This means in particular, that the junctions are located at the points $b_{0}, b_{i}$ and $-b_{i}$ for $i \in\{1, \ldots, K\}$. We have the following symmetry result:

Lemma 4.2. Assume that $\mu_{n}=\mu_{p}=1$ and that $N$ satisfies (4.1) and (4.27).

Then, for all $I$ (resp. $V)$ in $\mathbb{R}$, every solution $\left(\psi, n, p, J_{n}\right)\left(\right.$ resp. $\left.\left(\psi, n, p, J_{n}, I\right)\right)$ of $(\mathrm{RCD})_{I}\left(\right.$ resp. $\left.(\mathrm{RVD})_{V}\right)$ satisfies

$$
\psi\left((-x)^{ \pm}\right)=-\psi\left(x^{\mp}\right), n\left((-x)^{ \pm}\right)=p\left(x^{\mp}\right), p\left((-x)^{ \pm}\right)=n\left(x^{\mp}\right) \quad \forall x \in \bar{\Omega},
$$

and,

$$
J_{n}=\frac{I}{2} .
$$

Proof. Let $I$ be such that there exists a weak solution $\left(\psi, n, p, J_{n}\right)$ of $(\mathrm{RCD})_{I}$. Using (4.4)-(4.6), and since $\mu_{n}=\mu_{p}=1$ holds, we derive

$$
\begin{aligned}
(n p)^{\prime}(x)=-2^{-1} I N(x)+2^{-1}\left(2 J_{n}-I\right)(n+p)(x) \forall x \in\left(b_{i},\right. & \left.b_{i+1}\right), \\
& \forall i \in\{0, \ldots, K\} .
\end{aligned}
$$

On the other hand, we deduce from (4.8) that $n p$ is continuous on $\bar{\Omega}$. This, together with the integration of the above equation from $x=b_{i}$ to $x=b_{i+1}$, gives

$$
\begin{array}{r}
(n p)\left(b_{i+1}\right)-(n p)\left(b_{i}\right)=-2^{-1} I \int_{b_{i}}^{b_{i+1}} N(t) d t+2^{-1}\left(2 J_{n}-I\right) \int_{b_{i}}^{b_{i+1}}(n+p)(t) d t \\
\forall i \in\{0, \ldots, K\} .
\end{array}
$$

Adding the above equations from $i=0$ to $i=K$ and using the fact that $N$ is odd and that $(n p)(-1)=(n p)(1)$, we get

$$
\left(2 J_{n}-I\right) \int_{0}^{1}(n+p)(t) d t=0
$$

which implies (4.29), since $n+p$ is strictly positive on $\Omega$. On the other hand, since (4.4) holds, $n-p$ is an odd function. We now prove that $n p$ is an even function. We proceed as follows:

Using (4.29) in (4.30), we deduce that

$$
(n p)(x)-(n p)(0)=-2^{-1} I \int_{0}^{x} N(t) d t \quad \forall x \in[0,1] .
$$

Since $N$ is odd, we deduce that

$$
(n p)(x)=(n p)(-x) \quad \forall x \in \bar{\Omega} .
$$


Now, since (4.4) holds, we have

$$
n\left(x^{ \pm}\right)=\frac{N\left(x^{ \pm}\right)+\sqrt{\left.N^{2}\left(x^{ \pm}\right)+4(n p)(x)\right)}}{2} \quad \forall x \in \bar{\Omega},
$$

and,

$$
p\left(x^{ \pm}\right)=\frac{-N\left(x^{ \pm}\right)+\sqrt{\left.N^{2}\left(x^{ \pm}\right)+4(n p)(x)\right)}}{2} \quad \forall x \in \bar{\Omega} .
$$

Since $N$ is odd, whereas $n p$ is even, we deduce that we have

$$
n\left((-x)^{ \pm}\right)=p\left(x^{\mp}\right), \quad p\left((-x)^{ \pm}\right)=n\left(x^{\mp}\right) \quad \forall x \in \bar{\Omega},
$$

We now prove that $\psi$ is an odd function. From (4.5), and since $\mu_{n}=1$, we get

$$
\psi^{\prime}(t)=n^{-1}(t)\left(n^{\prime}(t)-I / 2\right) \quad \forall t \in\left(b_{i}, b_{i+1}\right), \quad \forall i \in\{0, \ldots, K\} .
$$

We integrate this last equation from $t=b_{i}$ to $t=x$ and use (4.31). This gives

$$
\psi\left(x^{-}\right)-\psi\left(b_{i}^{+}\right)=\int_{-b_{i}}^{-x} p^{-1}(s)\left(p^{\prime}(s)+I / 2\right) d s \quad \forall x \in\left(b_{i}, b_{i+1}\right], \quad \forall i \in\{0, \ldots, K\} .
$$

Now, since (4.6) holds on each subinterval $\left[-b_{i},-b_{i+1}\right]$, we obtain, using the above equation

$$
\psi\left(x^{-}\right)-\psi\left(b_{i}^{+}\right)=\psi\left(\left(-b_{i}\right)^{-}\right)-\psi\left((-x)^{+}\right) \quad \forall x \in\left(b_{i}, b_{i+1}\right], \quad \forall i \in\{0, \ldots, K\} .
$$

Using now the second equality of (4.8) and setting $x=b_{i}$ in (4.31), we deduce that

$$
\psi\left(\left(-b_{i}\right)^{-}\right)+\psi\left(b_{i}^{+}\right)=\psi\left(\left(-b_{i}\right)^{+}\right)+\psi\left(b_{i}^{-}\right) .
$$

Setting $i=K$ and $x=1$ in (4.32), and using (4.2), we obtain

$$
\psi\left(b_{K}^{+}\right)=-\psi\left(\left(-b_{K}\right)^{-}\right) .
$$

Using this last relation in (4.33), we get

$$
\psi\left(b_{K}^{-}\right)=-\psi\left(\left(-b_{K}\right)^{+}\right) .
$$

Using a backward induction on $i \in\{0, \ldots, K\}$, we deduce in the same way that

$$
\psi\left(b_{i}^{ \pm}\right)=-\psi\left(\left(-b_{i}\right)^{\mp}\right) \quad \forall i \in\{0, \ldots, K\} .
$$

These last equations, together with (4.32), imply that $\psi$ is an odd function. Hence, we proved that $\left(\psi, n, p, J_{n}\right)$ satisfies (4.28)-(4.29). This implies that, for all $V$, every solution $\left(\psi, n, p, J_{n}, I\right)$ of $(\mathrm{RVD})_{V}$ also satisfies (4.28)-(4.29) and concludes the proof of the lemma.

From this lemma, we obtain easily the following corollary:

Corollary 4.3. Assume that the hypotheses of Lemma 4.2 hold. Then, for every $V,\left(\psi, n, p, J_{n}, I\right)$ is a solution of $(\mathrm{RVD})_{V}$, if and only if $2 J_{n}=I$ and

$$
\begin{gathered}
n-p-N=0 \text { in } O=\bigcup_{i=0}^{i=K}\left(b_{i}, b_{i+1}\right), \\
\left(n^{\prime}-n \psi^{\prime}\right)=I / 2 \text { in } O \\
\left(p^{\prime}+p \psi^{\prime}\right)=-I / 2 \text { in } O \\
\psi(1)=\psi_{1}, n(1)=n_{1}, p(1)=p_{1}
\end{gathered}
$$




$$
\begin{aligned}
& {[n \exp (-\psi)]_{b_{i}}=[p \exp (\psi)]_{b_{i}}=0 \forall i \in\{1, \ldots, K\},} \\
& n(0) \exp (-\psi(0))=p(0) \exp (\psi(0))
\end{aligned}
$$

We denote system (4.34)-(4.38) by (SRVD) $V$. We can now describe, in the case of symmetric devices, the asymptotic behavior of the reduced currents as $|V|$ goes to infinity. In order to simplify the presentation of the results, we assume, in addition to (4.1) and (4.27), that $N$ satisfies

$$
\begin{gathered}
K \geq 1, N(x) \neq 0 \quad \forall x \in\left[b_{i}, b_{i+1}\right], \quad \forall i \in\{0, \ldots, K\}, \\
N>0 \quad \text { on }\left[b_{K}, 1\right] .
\end{gathered}
$$

Remark. The assumption (4.40) guarantees that the device contains an ohmic contact of each type ( $n$ and $p$ ), and that it is under forward (resp. reverse) bias, when $V>0$ (resp. $V<0$ ) holds. If $(4.40)$ is no longer assumed to hold, then there are no reverse saturation currents if the set $L^{\prime}$ defined in the following Theorem 4.4 is empty. The case $K=0$ corresponds to the case of symmetric $p n$ junctions. It has been analyzed in [12] for the case of a piecewise constant doping profile $N$. It can be shown easily that if $K=0$ and (4.40) hold, then the conclusions of Theorem 4.4 and Corollary 4.5 still hold. Therefore the assumption $K \geq 1$ is not restrictive.

Theorem 4.4. Assume that $N$ satisfies (4.1), (4.27), (4.39)-(4.40), that $\mu_{n}=$ $\mu_{p}=1$, and let $\left(\psi, n, p, J_{n}, I\right)$ be a solution of $(\mathrm{RVD})_{V}$. Then, the following properties hold:

- If $N$ satisfies

$$
0 \leq \int_{b_{i}}^{1} N(t) d t \quad \forall i \in\{0, \ldots, K-1\},
$$

then we have

$$
I \rightarrow+\infty \Longleftrightarrow V \rightarrow+\infty, \quad I \sim 2 V^{2}\left(\int_{0}^{1}\left(\int_{t}^{1} N(s) d s\right)^{-1 / 2} d t\right)^{-2} \text { as } V \rightarrow+\infty .
$$

- If (4.41) does not hold, then for all $V \geq 0$, we have

$$
I<I_{s, f}=2 \delta^{4} \min _{i \in L}\left(\left(-\int_{b_{i}}^{1} N(t) d t\right)^{-1}\right), I \rightarrow I_{s, f} \text { as } V \rightarrow+\infty,
$$

where

$$
L=\left\{i \in\{0, \ldots, K-1\}, \int_{b_{i}}^{1} N(t) d t<0\right\} .
$$

- Moreover, for all $V \leq 0$, we have

$$
I>I_{s, r}=2 \delta^{4} \max _{i \in L^{\prime}}\left(\left(-\int_{b_{i}}^{1} N(t) d t\right)^{-1}\right), I \rightarrow I_{s, r} \text { as } V \rightarrow-\infty,
$$

where

$$
L^{\prime}=\left\{i \in\{0, \ldots, K\}, \int_{b_{i}}^{1} N(t) d t>0\right\} \neq \emptyset
$$


Proof. Let $\left(\psi, n, p, J_{n}, I\right)$ be an arbitrary solution of $(\mathrm{RVD})_{V}$. Of course, $(\psi, n, p$, $\left.J_{n}, I\right)$ is an admissible solution of (RVD) $V$, if and only if $n$ and $p$ are strictly positive on $\bar{\Omega}$. Since we have $n+p=\sqrt{(n-p)^{2}+4 n p}$ and since $n-p=N$ hold, we deduce that $\left(\psi, n, p, J_{n}, I\right)$ is an admissible solution of $(\mathrm{RVD})_{V}$, if and only if $n p>0$ on $\bar{\Omega}$.

We deduce from Corollary 4.3 , that $\left(\psi, n, p, J_{n}, I\right)$ is a solution of (SRVD $)_{V}$. In particular, we deduce that

$$
(n p)(x)=2^{-1} I \int_{x}^{1} N(t) d t+\delta^{4} \quad \forall x \in \bar{\Omega} .
$$

Since (4.39) holds and since $N$ is continuous on each subinterval $\left[b_{i}, b_{i+1}\right], N$ keeps a constant sign on $\left[b_{i}, b_{i+1}\right]$. Hence, we have

$$
\begin{gathered}
\min \left((n p)\left(b_{i}\right),(n p)\left(b_{i+1}\right)\right) \leq(n p)(x) \leq \max \left((n p)\left(b_{i}\right),(n p)\left(b_{i+1}\right)\right), \\
\forall x \in\left[b_{i}, b_{i+1}\right], \forall i \in\{0, \ldots, K\} .
\end{gathered}
$$

Therefore, $n p$ is strictly positive on $\bar{\Omega}$, if and only if $(n p)\left(b_{i}\right)>0$ for all $i \in$ $\{0, \ldots, K\}$.

We now first consider the case where $V \geq 0$ holds. From (4.3), we deduce that $I \geq 0$ holds. If (4.41) holds, we deduce from (4.47)-(4.48) that $n p$ is strictly positive on $\bar{\Omega}$. Using now (4.4), we derive

$$
(n+p)\left(x^{ \pm}\right)=\sqrt{N^{2}\left(x^{ \pm}\right)+2 I \int_{x}^{1} N(t) d t+4 \delta^{4}} \quad \forall x \in[0,1] .
$$

From this and (4.34)-(4.36), we deduce, after integration, that

$$
\psi(1)-\psi(0)-\sum_{i=1}^{i=K}[\psi]_{b_{i}}=\sum_{i=0}^{i=K} \int_{b_{i}}^{b_{i+1}}\left(N^{\prime}(t)-I\right)\left(N^{2}(t)+2 I \int_{t}^{1} N(s) d s+4 \delta^{4}\right)^{-1 / 2} d t .
$$

Using now (1.10) in this last equation, we obtain

$$
\begin{aligned}
V= & \sum_{i=0}^{i=K} \int_{b_{i}}^{b_{i+1}}\left(I-N^{\prime}(t)\right)\left(N^{2}(t)+2 I \int_{t}^{1} N(s) d s+4 \delta^{4}\right)^{-1 / 2} d t-\psi(0) \\
& -\sum_{i=1}^{i=K}[\psi]_{b_{i}}+\log \left(\frac{N(1)+\left(N^{2}(1)+4 \delta^{4}\right)^{1 / 2}}{2 \delta^{2}}\right) .
\end{aligned}
$$

On the other hand, we deduce from the interface conditions (4.38), that

$$
[\psi]_{b_{i}}=\log \left(\frac{n\left(b_{i}^{+}\right)}{n\left(b_{i}^{-}\right)}\right) \quad \forall i \in\{1, \ldots, K\}
$$

and,

$$
\psi(0)=2^{-1} \log \left(\frac{n(0)}{p(0)}\right) .
$$

Using now (4.4), (4.47) and (4.49) in the above expressions for $[\psi]_{b_{i}}$ and $\psi(0)$, we obtain 


$$
\begin{gathered}
{[\psi]_{b_{i}}=\log \left(\frac{N\left(b_{i}^{+}\right)+\left(N^{2}\left(b_{i}^{+}\right)+2 I \int_{b_{i}}^{1} N(s) d s+4 \delta^{4}\right)^{1 / 2}}{N\left(b_{i}^{-}\right)+\left(N^{2}\left(b_{i}^{-}\right)+2 I \int_{b-i}^{1} N(s) d s+4 \delta^{4}\right)^{1 / 2}}\right)} \\
\forall i \in\{1, \ldots, K\}, \\
\psi(0)=2^{-1} \log \left(\frac{N\left(0^{+}\right)+\left(N^{2}\left(0^{+}\right)+2 I \int_{0}^{1} N(s) d s+4 \delta^{4}\right)^{1 / 2}}{-N\left(0^{+}\right)+\left(N^{2}\left(0^{+}\right)+2 I \int_{0}^{1} N(s) d s+4 \delta^{4}\right)^{1 / 2}}\right) .
\end{gathered}
$$

The relation (4.50) together with (4.51) and (4.52) lead to an implicit relation for $I$. From this implicit relation and since $N$ satisfies (4.39), we get (4.42).

Assume now that (4.41) does not hold and let $L$ be defined by (4.44). Then, we have $(n p)\left(b_{i}\right)>0$ for all $i \in\{0, \ldots, K\}-L$, whereas $(n p)\left(b_{i}\right)>0$ for $i \in L$ if and only if $I$ satisfies

$$
I<I_{s, f} .
$$

Moreover, since the equations (4.50)-(4.52) is still valid in this case, we deduce that (4.43) holds.

Assume now that $V \leq 0$ holds. Then, we deduce from (4.3) that $I \leq 0$ holds. Moreover, since $N$ satisfies (4.40), we deduce that the set $L^{\prime}$ defined by (4.46) is not empty. The proof of (4.45) is similar to that of (4.43) and is left to the reader. This concludes the proof of the theorem.

Remark. The expressions $I_{s, f}$ and $I_{s, r}$ give the saturation currents. From the above theorem, we know that if (4.43) does not hold, then the reduced currents saturate. One can easily check, that in this case the reduced electric field at the contacts also saturate, contrarily to what occurs for the full electric field. This saturation phenomenon is due to the loss of the Poisson's equation (1.1), so that the structural properties which lead to the Existence Theorem 2.7 (for every $I$ ) for the full problem $(\mathrm{CD})_{I}$ are lost, when one considers the reduced problem $(\mathrm{RCD})_{I}$. We can also remark that in the symmetric forward biased case, there exists only one saturation current, namely $I_{s, f}$. In [15] and [16], two different saturation currents have been obtained numerically for certain values of the doping profile $N$, in the case of nonsymmetric devices.

Theorem 4.4, together with Lemmas 3.2, 3.9 lead to the following corollary, which shows that, in general, the quasi-neutral approximation is not accurate for large $|V|$.

Corollary 4.5. Assume that $N$ satisfies the hypotheses of Theorem 4.4. Then, for any solution $\left(\psi, n, p, J_{n}, I\right)$ and $\left.\left(\bar{\psi}, \bar{n}, \bar{p}, \overline{J_{n}}, \bar{I}\right)\right)$ of $(\mathrm{VD})_{V}$ and of $(\mathrm{RVD})_{V}$ respectively, we have

$$
|I-\bar{I}| \rightarrow+\infty \quad \text { as }|V| \rightarrow+\infty
$$

\section{Conclusion And extensions}

We proved, in the course of this paper, that the one-dimensional drift-diffusion models possess specific structural properties, which are independent on the type of semiconductor devices under consideration. These properties allowed us, in turn, to prove several qualitative properties of these models related to existence, unique- 
ness, regularity of the solutions, nature of the (possible) bifurcation points of the voltage current characteristic, asymptotic behavior, monotonicity ... Moreover, by comparing the full and the reduced drift diffusion models, we showed that, in general, the asymptotic behavior of the total currents predicted by the reduced model, is inaccurate for the full problem. This proves that, in general, the assumption of electroneutrality should not be considered for large voltages.

The main results of this paper can be easily generalized, with minor changes, to the case of arbitrary boundary values $\psi_{ \pm 1}, n_{ \pm 1}>0, p_{ \pm 1}>0$, such as the ones which are under consideration in electrochemistry.

When the generation recombination term $R$ is not zero, the current driven model consists of equations (1.1)-(1.3), (5.1) subject to the boundary conditions (1.5)(1.7), where the equation

$$
J_{n}^{\prime}=R,
$$

replaces equation (1.4). There exist various expressions for $R$ corresponding to different physical mechanisms (see e.g. [11]). Generally $R$ depends on the space variable and on the unknowns $\psi, n, p$ (as is the case for Shockley-Read-Hall and Auger generation recombination terms) and eventually on $J_{n}$ (as is the case for the impact ionization term). We denote by $(\mathrm{GCD})_{I}$ the current driven model corresponding to the case of a nonzero generation recombination term. The existence and uniqueness result for $(\mathrm{CD})_{I}$ given in section 2 , uses the fact that when $R=0$, $J_{n}$ is a constant. This is no longer true when $R$ is not identically zero, so that the techniques of Theorems 2.1 and 2.7 are no longer applicable. However, we can extend the results of section 2 to the case of small generation recombination term.

We proceed as follows. We introduce the following spaces equipped with their usual norms

$$
X=\left(H^{2}(\Omega)\right)^{3} \times H^{1}(\Omega) \quad, \quad Y=L^{2}(\Omega) \times\left(H^{1}(\Omega)\right)^{2} \times L^{2}(\Omega) \times \mathbb{R},
$$

and we set $W=\left(\psi, n, p, J_{n}\right)$.

We assume that $R$ satisfies

$$
R(x, W)=\tau S(x, W) \quad \forall x \in \bar{\Omega}, \quad \forall W \in X,
$$

where $\tau$ is a small real parameter and where $S(x,$.$) belongs to \mathcal{C}^{2}\left(\mathbb{R} \times(0,+\infty)^{2} \times \mathbb{R}\right)$ for all $x$ in $\Omega$ and $\partial_{W}^{\alpha} S(., W)$ belongs to $L^{\infty}(\Omega)$ uniformly for $W$ in bounded sets of $\mathbb{R} \times(0,+\infty)^{2} \times \mathbb{R}$, and for $\alpha=0,1,2$.

For each fixed $I,(\mathrm{GCD})_{I}$ can be written under the form

$$
\mathcal{F}_{I}(\tau, W)=0 .
$$

We can now give the following local result

Theorem 5.1. Assume that $N$ is in $L^{\infty}(\Omega)$, that $\mu_{n}, \mu_{p}$ are strictly positive constants, and that $R$ satisfies the assumptions given above. Then for every $I$ in $\mathbb{R}$, there exists $\tau_{0}>0$ such that $(\mathrm{GCD})_{I}$ has locally a unique solution in $X$ for every $|\tau|<\tau_{0}$.

Proof. It is easy to check that the assumptions on $R$ imply that $\mathcal{F}_{I}$ is a continuously Fréchet differentiable map. From Corollary 2.17, we know that (5.2) admits a unique solution $W_{0}$ in $\left(H^{2}(\Omega)\right)^{3} \times \mathbb{R}$ for $\tau=0$. By using a proof similar to that of Theorem 2.1, we show that the linearized operator $\partial_{W} \mathcal{F}_{I}\left(0, W_{0}\right)$ is one-to-one. Moreover, it is easy to check that this operator is a Fredholm operator of index 0. This, together with the implicit function theorem, concludes the proof. 
The generalization of these results to the case of nonsmall generation recombination terms is not trivial and requires probably more refined techniques. The multi-dimensional case should also be analyzed. The Existence Theorem 2.7 is given for constant mobilities $\mu_{n}, \mu_{p}$. We conjecture that this theorem is still valid in the case of space dependent mobilities which are uniformly bounded away from zero. The difficulty in that case, is that we do not have the a priori estimate given in

Lemma 2.8. The impact of the physical scales on certain mathematical properties of the solutions (as is done for instance when the fact that $\varepsilon$ is small is taken into consideration) should also be analyzed.

We do not consider in this paper the problem of uniqueness or multiplicity of the solutions of $(\mathrm{VD})_{V}$. Contrary to the results obtained in this paper, the answer to this problem highly depends on the type of semiconductor devices under consideration through the doping profile $N$ and requires other techniques (see [5], [2], [1]). Nevertheless, the monotonicity lemmas given in this paper are particularly useful for this open question and have already been used under a much weaker form in the above-mentioned papers.

\section{ACKNOWLEDGMENT}

The author would like to thank Professor Joel Smoller for fruitful discussions and invaluable encouragement.

\section{REFERENCES}

1. F. Alabau. New uniqueness theorems for the one-dimensional drift-diffusion semiconductor device equations. Siam J. Math. Anal., 26:715-737, 1995.

2. F. Alabau. A uniqueness theorem for reverse-biased diodes. To appear in Applicable Anal.

3. F. Alabau. Analyse asymptotique et simulation numérique des équations des semiconducteurs. PhD thesis, Université Paris 6, 1987.

4. F. Alabau. Uniform asymptotic error estimates for the semiconductor device and electrochemistry equations. Nonlinear Anal., 14:123-139, 1990. MR 92i:34068

5. F. Alabau. A method for proving uniqueness theorems for the stationary semiconductor device and electrochemistry equations. Nonlinear Anal., 18:861-872, 1992. MR 93d:78006

6. F. Alabau. Etude des modèles de dérive-diffusion à courant donné et potentiel donné dans le cas des semi-conducteurs mono-dimensionnels. C.R. Acad. Sci., 316:885-890, 1993. MR 94b:78016

7. E.A. Coddington and N. Levinson. Theory of ordinary differential equations. McGraw-Hill, New York, 1955. MR 16:1022b

8. D. Gilbarg and N.S. Trudinger. Elliptic partial differential equations of second order. 2nd ed. Springer-Verlag, Berlin, 1983. MR 86c:35035

9. J. Henry and B. Louro. Singular perturbation theory applied to electrochemistry equations in the case of electroneutrality. Nonlinear Anal., 13:787-801, 1989. MR 90g:35156

10. T. Kerkhoven. On the one-dimensional current driven semiconductor equations. Siam J. Appl. Math., 51:748-774, 1991. MR 91m:35223

11. P.A. Markowich. The stationary semiconductor device equations. Springer, Wien-New York, 1986. MR 87b:78042

12. P.A. Markowich and C. Schmeiser. Uniform asymptotic representation of the basic semiconductor device equations. IMA J. Appl. Math., 36:43-57, 1986. MR 90e:94049

13. M.S. Mock. An example of nonuniqueness of stationary solutions in semiconductor device models. Compel, 1:165-174, 1982.

14. W.V. Van Roosbroeck. Theory of flow of electrons and holes in germanium and other semiconductors. Bell Syst. Techn. J., 29:560-607, 1950.

15. I. Rubinstein. Electro-diffusion of ions. Siam Studies in Applied Mathematics, 1990. MR 91m:78019 
16. H. Steinruck. A bifurcation analysis of the one-dimensional steady-state semiconductor device equations. Siam J. Appl. Math., 49:1102-1121, 1989. MR 90h:94066

17. M.J. Ward, L.G. Reyna, and F.M. Odeh. Multiple steady state solutions in a multijunction semiconductor device. Siam J. Appl. Math., 51:90-123, 1991. MR 91m:78020

CeRemaB, Université Bordeaux I, 351, cours de la Libération 33405 Talence Cedex, FRANCE

E-mail address: alabau@math.u-bordeaux.fr 